\title{
Non-Glimm-Effros equivalence relations at second projective level
}

\author{
by
}

\author{
Vladimir Kanovei (Moscow)
}

\begin{abstract}
A model is presented in which the $\Sigma_{2}^{1}$ equivalence relation $x \mathrm{C} y$ iff $\mathrm{L}[x]=$ $\mathrm{L}[y]$ of equiconstructibility of reals does not admit a reasonable form of the Glimm-Effros theorem. The model is a kind of iterated Sacks generic extension of the constructible model, but with an "ill" founded "length" of the iteration. In another model of this type, we get an example of a $\Pi_{2}^{1}$ non-Glimm-Effros equivalence relation on reals. As a more elementary application of the technique of "ill" founded Sacks iterations, we obtain a model in which every nonconstructible real codes a collapse of a given cardinal $\kappa \geq \aleph_{2}^{\text {old }}$ to $\aleph_{1}^{\text {old }}$.
\end{abstract}

Introduction. Theorems of the following type are quite usual in mathematics:

Every object in some domain is either "regular" in some specified sense, or, if it is "singular" then it includes a certain distinguished "singular" object.

For instance, by Suslin's old theorem, a Borel, or, more generally, $\boldsymbol{\Sigma}_{1}^{1}$ set of reals is either countable (= "regular") or contains a perfect subset (= the distinguished type of uncountable sets).

The behaviour of more complicated sets with respect to this particular "dichotomy" was completely investigated in the early era of forcing: first, a $\boldsymbol{\Sigma}_{2}^{1}$ set is either of cardinality $\leq \aleph_{1}$ or contains a perfect subset; second, nothing like this can be proved for $\Pi_{2}^{1}$ sets unless we use special strong axioms (like the axiom of determinacy) or work in special "regular" models (for example the Solovay model).

It is a related but more general and much more difficult problem to investigate, in this manner, the number of equivalence classes of an equiv-

1991 Mathematics Subject Classification: Primary 03E15, 03E35, 04A15; Secondary $03 \mathrm{E} 40$.

Partially supported by the Max Planck Institute (Bonn), University of Wuppertal, and IPM (Tehran) during visits in 1995-1996, AMS and DFG, and NIOKR MPS. 
alence relation on the reals. This problem can be traced back (at least) to the origins of descriptive set theory $\left({ }^{1}\right)$.

It was in the 1970's that Silver [17] proved that a $\boldsymbol{\Pi}_{1}^{1}$ equivalence relation on the reals either has countably many equivalence classes or admits a perfect set of pairwise inequivalent reals. (The Suslin theorem is an easy corollary: indeed, if $X$ is a $\boldsymbol{\Sigma}_{1}^{1}$ set of reals then the equivalence $\mathrm{E}$ defined as equality on $X$ and $x \mathrm{E} y$ for all $x, y \notin X$, is $\Pi_{1}^{1}$.)

Moreover, it was recently recognized that equivalence relations allow a different type of investigation, related to enumeration of classes by sets of ordinals (e.g. reals) rather than ordinals themselves. Harrington, Kechris, and Louveau [5] proved that each Borel equivalence relation $\mathrm{E}$ on the reals satisfies one and only one of the following conditions:

(I) E admits a Borel enumeration of the equivalence classes by reals.

(II) $\mathrm{E}_{0}$, the Vitali equivalence, is continuously embedded in $\mathrm{E}\left({ }^{2}\right)$.

Notation. An enumeration of classes for an equivalence relation $\mathrm{E}$ on the reals is a function $U$ defined on the reals and satisfying $x \mathrm{E} y$ iff $U(x)=$ $U(y)$ for all $x, y . \mathrm{E}_{0}$ is the Vitali equivalence on the Cantor space $\mathcal{D}=2^{\omega}$, defined by: $x \mathrm{E}_{0} y$ iff $x(n)=y(n)$ for all but finitely many $n \in \omega$. An embedding of $\mathrm{E}_{0}$ into $\mathrm{E}$ is a 1-1 function $U: \mathcal{D} \rightarrow$ reals such that we have $x \mathrm{E}_{0} y \Leftrightarrow U(x) \mathrm{E} U(y)$ for all $x, y \in \mathcal{D}$.

The dichotomy (I) vs. (II) was called the Glimm-Effros dichotomy in [5] as Glimm and Effos proved some particular results. (We refer the reader to [5] as the basic source of information on the history of this type of theorems, to Hjorth and Kechris [9] and Kechris [13] for a review of further development, to all the three for applications and related topics, and to Kechris [14] for broad references on the subject.)

Theorems of this type, but with a weaker condition (I) $\left(^{3}\right)$, are known for $\Sigma_{1}^{1}$ equivalence relations, provided either the universe satisfies the sharps hy-

$\left({ }^{1}\right)$ Luzin pointed out in [15] that, although it looks natural that the Vitali equivalence on the reals has continuum-many equivalence classes, a concrete enumeration of the equivalence classes by reals had not been known. (In the absence of the axiom of choice the Vitali equivalence can have strictly more equivalence classes than the cardinality of continuum, see Kanovei [10].) Even earlier Sierpiński [16] demonstrated that if the set of all Vitali classes can be linearly ordered then there exists a nonmeasurable set of reals, having approximately the same projective class as the linear order, provided it is projective. The Vitali equivalence in general plays a special role in modern investigations.

$\left({ }^{2}\right)$ Relations satisfying (I) are called smooth. Notice that $\mathrm{E}_{0}$ is not smooth.

$\left({ }^{3}\right) \Delta_{1}^{\text {HC }}$ enumeration of the equivalence classes by countable (of any length $<\omega_{1}$ ) binary sequences. 
pothesis (Hjorth and Kechris [9]) or each real belongs to a generic extension of the constructible universe L (Kanovei $[12])\left({ }^{4}\right)$.

We prove (this is the main result of this article) that the picture changes at the second projective level: the classes $\Sigma_{2}^{1}$ and $\Pi_{2}^{1}$ contain counterexamples, equivalence relations which do not admit a (provable in ZFC) theorem of the Glimm-Effros type, at least in the domain of real-ordinal definable (R-OD, in brief) enumerations and embeddings.

THEOREM 1. It is consistent with $\mathbf{Z F C}$ that the $\Sigma_{2}^{1}$ equivalence relation C of "equiconstructibility", defined on the reals by $x \mathrm{C} y$ iff $\mathrm{L}[x]=\mathrm{L}[y]$, has $\mathfrak{c}$ equivalence classes, and:

- neither C has a R-OD enumeration of the equivalence classes by sets of ordinals,

- nor C admits a R-OD pairwise C-inequivalent set of cardinality $\mathfrak{c}$,

and in addition either of the following two cardinal equalities can be modelled in the universe: $\mathfrak{c}=\aleph_{1}=\aleph_{1}^{\mathrm{L}}$ or $\mathfrak{c}=\aleph_{2}=\aleph_{2}^{\mathrm{L}}$.

THEOREM 2. It is consistent with $\mathbf{Z F C}$ that some $\Pi_{2}^{1}$ equivalence relation $\mathrm{E}$ on the reals has $\mathfrak{c}$ equivalence classes, and:

- neither $\mathrm{E}$ has a R-OD enumeration of the equivalence classes by sets of ordinals,

- nor $\mathrm{E}_{0}$ is embedded in $\mathrm{E}$ via a R-OD embedding,

and in addition either of the following two cardinal equalities can be modelled in the universe: $\mathfrak{c}=\aleph_{1}=\aleph_{1}^{\mathrm{L}}$ or $\mathfrak{c}=\aleph_{2}=\aleph_{2}^{\mathrm{L}}$.

Remarks. The "nor" assertion of Theorem 1 implies the "nor" assertion of Theorem 2, because obviously there exists a perfect set of pairwise $\mathrm{E}_{0}$-inequivalent points. It is not known whether one can strengthen the "nor" assertion of Theorem 2 to the form of Theorem 1 or at least to the form of the non-existence of a $\mathrm{R}$-OD reduction of $\mathrm{E}_{0}$ to $\mathrm{C}$. (A reduction is the same as an embedding but not necessarily 1-1.) The equivalence relation we define for Theorem 2 does not seem to lead to this goal. (See footnote 11 in Section 6.)

It makes no sense to look for non-R-OD enumerations, assuming we work in ZFC (with Choice). Equally it would be silly to look for enumerations by sets of sets of ordinals (the next level) because each equivalence class is an object of this type.

The theorems are close to possible optimal counterexamples. Indeed, Hjorth [8] proved that every $\boldsymbol{\Delta}_{2}^{1}$ equivalence relation (more generally, a

$\left({ }^{4}\right)$ Friedman and Velickovic [2], Hjorth [7, 8], Kanovei [11] obtained partial results of this type for $\boldsymbol{\Sigma}_{1}^{1}, \boldsymbol{\Pi}_{1}^{1}$, and more complicated relations, and different relevant theorems on equivalence relations, which we do not intend to discuss in detail. 
relation which is both $\omega_{1}$-Suslin and co- $\omega_{1}$-Suslin) which has the property that the equality of the $\boldsymbol{\Sigma}_{2}^{1}$ and $\boldsymbol{\Pi}_{2}^{1}$ definitions is preserved in Cohen generic extensions, admits a Glimm-Effros theorem, with an enumeration of classes by $\omega_{1}$-long binary sequences in (I).

It is a very interesting problem at the moment to figure out whether all $\boldsymbol{\Delta}_{2}^{1}$ relations admit a reasonable dichotomy theorem of the "Glimm-Effros" type. (Since the models we construct for the theorems are very special, perhaps even the classes $\boldsymbol{\Sigma}_{2}^{1}$ and $\boldsymbol{\Pi}_{2}^{1}$ admit a Glimm-Effros dichotomy under certain reasonably weak assumptions.)

Another problem is to obtain counterexamples consistent with $\mathfrak{c}>\aleph_{2}$. The technique employed in the proofs of Theorems 1 and 2 does not work.

The models. The proposed models for Theorems 1 and 2 are iterated Sacks extensions of the constructible model, having a nonwellordered set as the "length" of iteration, hence not a kind of iterated generic models in the usual setting (see Baumgartner and Laver [1], Groszek and Jech [4] on iterations of the Sacks forcing), where the length of the iteration is, by definition, an ordinal, or at least a wellfounded set. We use "ill" ordered and even "ill" founded Sacks iterations to prove the theorems.

A construction of iterated Sacks generic extensions having inverse ordinals as the "length" of iteration was given by Groszek [3]. We make different technical arrangements to obtain "ill" ordered and even "ill" founded Sacks iterations. (The model for Theorem 2 is an "ill" founded nonlinear iteration; a model for Theorem 1 can be obtained in two different ways: as a linear "ill" ordered Sacks iteration, and as a nonlinear wellfounded Sacks iteration; the latter version is equivalent to the usual countable support iteration of the product Sacks $\times$ Sacks forcing, of length $\omega_{1}$ or $\omega_{2}$.)

Let $\mathbf{I}$ be a partially ordered set in $\mathfrak{M}$, the ground model, — the intended "length" of the iteration. A typical forcing condition for the notion of forcing leading to Sacks iterations of length $\mathbf{I}$ is, in $\mathfrak{M}$, a set $X \subseteq \mathcal{D}^{\zeta}$, where $\zeta \subseteq \mathbf{I}$ is countable while $\mathcal{D}=2^{\omega}$, of the form $X=H^{\prime \prime} \mathcal{D}^{\zeta}$, where $H$ is a $1-1$ continuous function such that

$$
x\lceil\xi=y\lceil\xi \Leftrightarrow H(x)\lceil\xi=H(y)\lceil\xi
$$

for all $x, y \in \mathcal{D}^{\zeta}$ and any initial segment $\xi$ of $\zeta$. Sets $X$ of this form may be called iterated perfect sets in $\mathcal{D}^{\zeta}$.

Section 1 contains the definition and several basic lemmas, mostly of simple "geometric" nature, related to the forcing conditions.

Section 2 shows how to split and assemble the forcing conditions via a kind of splitting technique, common for different forms of the Sacks forcing.

Section 3 ends the study of the forcing conditions by a theorem specifying the behaviour of continuous functions mapping the conditions into the reals. 
Sections 4 and 5 define and study the extensions. We prove that the forcing notion associated with a partially ordered set $\mathbf{I}$ in the ground model $\mathfrak{M}$ produces generic models of the form $\mathfrak{N}=\mathfrak{M}\left[\left\langle\mathbf{a}_{i}: i \in \mathbf{I}\right\rangle\right]$, where each $\mathbf{a}_{i} \in \mathcal{D}$ is Sacks generic over the model $\mathfrak{M}\left[\left\langle\mathbf{a}_{j}: j<i\right\rangle\right]$, —a property which witnesses that $\mathfrak{N}$ is a kind of iterated Sacks extension of $\mathfrak{M}$ despite the fact that $\mathbf{I}$ may not be wellordered. (A closer inspection, out of the scope of this paper, shows that in the case when $\mathbf{I}$ is an ordinal, the models we obtain are usual countable support iterated Sacks extensions of the ground model.)

We prove a cardinal preservation theorem, and a very important theorem which says that each real in $\mathfrak{N}$ can be obtained by applying a continuous function coded in $\mathfrak{M}$ to a countable sequence of generic reals. This theorem allows us to transform properties of continuous functions in the ground model to properties of reals in the extension.

In particular, it turns out (Section 5) that if every initial segment of $\mathbf{I}$ belongs to $\mathfrak{M}$ then the degrees of $\mathfrak{M}$-constructibility of reals in the extension are in 1-1 correspondence with countably cofinal initial segments of $\mathbf{I}$.

The proof of Theorem 1 in Section 6 utilizes a particular property of the sets $\mathbf{I}=\left(\omega_{1}\right.$ or $\left.\omega_{2}\right) \times \mathbb{Z}$, where $\mathbb{Z}=\{\ldots,-2,-1,0,1,2, \ldots\}$, the integers: each copy of $\mathbb{Z}$ admits nontrivial order automorphisms, shiftings. This does not allow a real in the extension to "know" definitely the exact place, say $\langle\alpha, z\rangle \in \omega_{1} \times \mathbb{Z}$, of its degree of constructibility. Another possibility is $\mathbf{I}=$ $\left(\omega_{1}\right.$ or $\left.\omega_{2}\right) \times($ unordered $\{0,1\})$, which is equivalent to the iteration of the forcing Sacks $\times$ Sacks, of length $\omega_{1}$ or $\omega_{2}$. We do not know whether an ordinal length iteration of the Sacks forcing can prove Theorem 1.

A modification, $\mathbf{I}=\left(\omega_{1}\right.$ or $\left.\omega_{2}\right) \times(\mathbb{Z} \times\{0,1\})$, is applied to prove Theorem 2. This case makes essential use of order automorphisms generated by "exchanges" $0 \leftrightarrow 1$. We do not know how to prove this theorem not involving "ill" founded Sacks iterations.

A model in which every nonconstructible real collapses $\aleph_{2}$ to $\aleph_{1}$. This is an easier application of the technique of "ill" founded Sacks iterations.

TheOREM 3. Let $\kappa>\aleph_{1}^{\mathfrak{M}}$ be a cardinal in a countable model $\mathfrak{M}=\mathrm{V}=$ $\mathrm{L}$. Then there exists a generic extension $\mathfrak{N}$ of $\mathfrak{M}$ in which $\aleph_{1}^{\mathfrak{M}}$ is still a cardinal, there are "new" reals, and $\operatorname{card} \kappa=\aleph_{1}^{\mathfrak{M}}$ in every submodel $\mathfrak{M}[r]$ where $r$ is a real in $\mathfrak{N} \backslash \mathfrak{M}$.

(It is clear that the result is impossible for the cardinal $\aleph_{0}$ instead of $\aleph_{1}$ since a collapse of an uncountable cardinal to $\aleph_{0}$ provides "new" reals which do not collapse cardinals, e.g. Cohen generic reals.)

Of course different forcing notions produce reals that code much more sophisticated things, but the model for Theorem 3 is somewhat exceptional because first it is really simple (we use, in Section 5, Sacks iterations of "length" equal to the inverted cardinal $\kappa$, and exploit the known phenom- 
ena that each next Sacks real "knows" the previous steps of the iteration, which compels every "new" real to code the collapse) and does not involve a complicated coding technique.

1. Iterated perfect sets. Let $\mathbf{C P O}$ be the class of all countable (including finite) partially ordered sets $\zeta=\langle\zeta ;<\rangle$. The Greek letters $\xi, \eta, \zeta, \vartheta$ will denote sets in CPO. The characters $i, j$ are used to denote elements of sets in CPO.

For any $\zeta \in \mathbf{C P O}, \mathbf{I S}_{\zeta}$ is the collection of all initial segments of $\zeta$. (A set $\xi \subseteq \zeta$ is an initial segment of $\zeta$ iff $i \in \xi$ implies $j \in \xi$ whenever $i, j \in \zeta$ and $j<i$.) For instance, $\emptyset$ and $\zeta$ itself belong to $\mathbf{I S}_{\zeta}$.

We shall usually have fixed a "basic" p.o. set $\zeta \in \mathbf{C P O}$, so that all other p.o. sets actually involved in the reasoning are subsets of $\zeta$. In this case, for any $i \in \zeta$ we consider initial segments $[<i]=\{j \in \zeta: j<i\}$ and $[\nsupseteq i]=\{j \in \zeta: j \nsupseteq i\}$, and $[\leq i],[\ngtr i]$ defined analogously.

We define $\mathcal{N}=\omega^{\omega}$, the Baire space; points of $\mathcal{N}$ will be called reals.

$\mathcal{D}=2^{\omega}$ is the Cantor space. For any countable set $\xi, \mathcal{D}^{\xi}$ is the product of $\xi$ copies of $\mathcal{D}$ with the product topology. Then every $\mathcal{D}^{\xi}$ is a compact space, homeomorphic to $\mathcal{D}$ itself unless $\xi=\emptyset$.

Assume that $\eta \subseteq \xi$. If $x \in \mathcal{D}^{\xi}$ then let $x\left\lceil\eta \in \mathcal{D}^{\eta}\right.$ denote the usual restriction. If $X \subseteq \mathcal{D}^{\xi}$ then let $X\lceil\eta=\{x\lceil\eta: x \in X\}$.

But if $Y \subseteq \mathcal{D}^{\eta}$ then we set $Y \uparrow^{-1} \xi=\left\{x \in \mathcal{D}^{\xi}: x\lceil\eta \in Y\}\right.$.

To save space, let $X \uparrow_{<i}$ mean $X \uparrow[<i], X \uparrow_{\nsupseteq i}$ mean $X \uparrow[\nsupseteq i]$, etc.

To describe the idea behind the definition of iterated perfect sets, recall that the Sacks forcing consists of perfect subsets of $\mathcal{D}$, i.e. sets of the form $H " \mathcal{D}=\{H(a): a \in \mathcal{D}\}$ where $H: \mathcal{D} \stackrel{\text { onto }}{\longrightarrow} X$ is a homeomorphism.

To get a product Sacks model, with two factors (the case of a two-element unordered set as the length of iteration) we have to consider sets $X \subseteq \mathcal{D}^{2}$ of the form $X=H^{\prime \prime} \mathcal{D}^{2}$ where $H$, a homeomorphism defined on $\mathcal{D}^{2}$, splits in an obvious way into a pair of one-dimensional homeomorphisms.

To get an iterated Sacks model, with two stages of iteration (the case of a two-element ordered set as the length of iteration) we have to consider sets $X \subseteq \mathcal{D}^{2}$ of the form $X=H$ " $\mathcal{D}^{2}$ where $H$, a homeomorphism defined on $\mathcal{D}^{2}$, satisfies the following: if $H\left(a_{1}, a_{2}\right)=\left\langle x_{1}, x_{2}\right\rangle$ and $H\left(a_{1}^{\prime}, a_{2}^{\prime}\right)=\left\langle x_{1}^{\prime}, x_{2}^{\prime}\right\rangle$ then $a_{1}=a_{1}^{\prime} \Leftrightarrow x_{1}=x_{1}^{\prime}$.

The general case results in the following definition.

Definition. For any $\zeta \in \mathbf{C P O}, \operatorname{Perf}_{\zeta}$ is the collection of all sets $X \subseteq \mathcal{D}^{\zeta}$ such that there is a homeomorphism $H: \mathcal{D}^{\zeta} \stackrel{\text { onto }}{\longrightarrow} X$ satisfying

$$
x_{0} \uparrow \xi=x_{1} \uparrow \xi \Leftrightarrow H\left(x_{0}\right)\left\lceil\xi=H\left(x_{1}\right)\lceil\xi\right.
$$

for all $x_{0}, x_{1} \in \operatorname{dom} H$ and $\xi \in \mathbf{I S}_{\zeta}$. Homeomorphisms $H$ satisfying this 
requirement will be called projection-keeping. So, sets in $\operatorname{Perf}_{\zeta}$ are images of $\mathcal{D}^{\zeta}$ via projection-keeping homeomorphisms.

If $H: \mathcal{D}^{\zeta} \stackrel{\text { onto }}{\longrightarrow} X$ is a projection-keeping homeomorphism then we define, for any $\xi \in \mathbf{I S}_{\zeta}$, an associated projection-keeping homeomorphism $H_{\xi}$ of $\mathcal{D}^{\xi}$ onto $X\left\lceil\xi\right.$ by $H_{\xi}(x \mid \xi)=H(x)\left\lceil\xi\right.$ for all $x \in \mathcal{D}^{\zeta}$.

Proposition 4. Every set $X \in \operatorname{Perf}_{\zeta}$ is closed and has the following properties:

P-1. If $i \in \zeta$ and $\left.z \in X\right|_{<i}$ then $D_{X z}(i)=\left\{x(i):\left.x \in X \& x\right|_{<i}=z\right\}$ is a perfect set in $\mathcal{D}$.

P-2. If $\xi \in \mathbf{I S}_{\zeta}$ and a set $X^{\prime} \subseteq X$ is open in $X$ (in the relative topology) then the projection $X^{\prime} \mid \xi$ is open in $X\lceil\xi$.

P-3. If $\xi, \eta \in \mathbf{I S}_{\zeta}, x \in X\lceil\xi, y \in X\lceil\eta$, and $x \uparrow(\xi \cap \eta)=y \uparrow(\xi \cap \eta)$, then $x \cup y \in X \uparrow(\xi \cup \eta)$.

(P-2 says that the projection from $X$ to $X\lceil\xi$ is an open map.)

This proposition could be taken as the base for an independent treatment of the notion; however, it is not true that the requirements $\mathrm{P}-1, \mathrm{P}-2, \mathrm{P}-3$ fully characterize $\operatorname{Perf}_{\zeta}$.

Pro of. Clearly $\mathcal{D}^{\zeta}$ satisfies P-1, P-2, P-3. Moreover, one easily shows that projection-keeping homeomorphisms preserve the requirements.

Let us prove several simple lemmas on forcing conditions.

The following lemma shows how P-3 works.

Lemma 5. Suppose that $X \in \operatorname{Perf}_{\zeta}, \xi, \eta \in \mathbf{I S}_{\zeta}, Y \subseteq X\lceil\eta$, and $Z=$ $X \cap\left(Y \uparrow^{-1} \zeta\right)$. Then $Z\left\lceil\xi=\left(X\lceil\xi) \cap\left(Y \uparrow(\xi \cap \eta) \uparrow^{-1} \xi\right)\right.\right.$.

Proof. The inclusion $\subseteq$ is quite easy. To prove the opposite direction let $x$ belong to the right-hand side. Then in particular $x \uparrow(\xi \cap \eta)=y \uparrow(\xi \cap \eta)$ for some $y \in Y$. On the other hand, $x \in X\lceil\xi$ and $y \in X\lceil\eta$. Property P-3 of $X$ (see Proposition 4) implies $x \cup y \in X \uparrow(\xi \cup \eta)$. Thus $x \cup y \in Z \uparrow(\xi \cup \eta)$ since $y \in Y \subseteq X\lceil\eta$, so $x \in Z\lceil\xi$.

Lemma 6. If $X \in \operatorname{Perf}_{\zeta}$ and $\xi \in \mathbf{I S}_{\zeta}$ then $X \mid \xi \in \operatorname{Perf}_{\xi}$.

Proof. If $X \in \operatorname{Perf}_{\zeta}$ via $H$ then $H_{\xi}$ witnesses $X\left\lceil\xi \in \operatorname{Perf}_{\xi}\right.$.

Lemma 7. Suppose that $H$ is a projection-keeping homeomorphism, defined on some $X \in \operatorname{Perf}_{\zeta}$. Then the image $H$ " $X=\{H(x): x \in X\}$ belongs to $\operatorname{Perf}_{\zeta}$.

Proof. A superposition of projection-keeping homeomorphisms is obviously a projection-keeping homeomorphism.

Lemma 8. If $X \in \operatorname{Perf}_{\zeta}$, a set $X^{\prime} \subseteq X$ is open in $X$, and $x_{0} \in X^{\prime}$, then there is a set $X^{\prime \prime} \in \operatorname{Perf}_{\zeta}, X^{\prime \prime} \subseteq X^{\prime}$, clopen in $X$ and containing $x_{0}$. 
Proof. By the previous lemma, it suffices to prove the result provided $X=\mathcal{D}^{\zeta}$. We observe that if $x_{0} \in X^{\prime} \subseteq \mathcal{D}^{\zeta}$ and $X^{\prime}$ is open in $\mathcal{D}^{\zeta}$ then there exists a basic clopen set $C \subseteq X^{\prime}$ containing $x_{0}$. (Basic clopen sets are sets of the form $C=\left\{x \in \mathcal{D}^{\zeta}: u_{1} \subset x\left(i_{1}\right) \& \ldots \& u_{m} \subset x\left(i_{m}\right)\right\}$, where $m \in \omega, i_{1}, \ldots, i_{m} \in \zeta$ are pairwise different, and $u_{1}, \ldots, u_{m} \in 2^{<\omega}$. One easily proves that every set $C$ of this type belongs to $\operatorname{Perf}_{\zeta}$.)

Lemma 9. Let $X, Y \in \operatorname{Perf}_{\zeta}$ and $\eta \in \mathbf{I S}_{\zeta}, X\lceil\eta=Y\lceil\eta$. There is a projection-keeping homeomorphism $H: X \stackrel{\text { onto }}{\longrightarrow} Y$ such that $H(x)\lceil\eta=x\lceil\eta$ for all $x \in X$.

Proof. Let $F: \mathcal{D}^{\zeta} \stackrel{\text { onto }}{\longrightarrow} X$ and $G: \mathcal{D}^{\zeta} \stackrel{\text { onto }}{\longrightarrow} Y$ witness that the sets $X$ and $Y$ resp. belong to $\operatorname{Perf}_{\zeta}$. Define $H(x)=G\left(G_{\eta}^{-1}\left(x\lceil\eta) \cup F^{-1}(x)\lceil(\zeta \backslash \eta))\right.\right.$ for all $x \in X$. Then $H$ " $X \subseteq Y$ by the choice of $G$. Let us prove that $H$ " $X=Y$. Let $y \in Y$. Set $x=F\left(F_{\eta}^{-1}\left(y\lceil\eta) \cup G^{-1}(y)\lceil(\zeta \backslash \eta))\right.\right.$ (the dual transform). Then we have $x\left\lceil\eta=y\left\lceil\eta\right.\right.$ while $F^{-1}(x)\left\lceil(\zeta \backslash \eta)=G^{-1}(y)\lceil(\zeta \backslash \eta)\right.$, so that $H(x)=G\left(G^{-1}(y)\right)=y$, as required.

Notice that $H(x)\left\lceil\eta=G_{\eta}\left(G_{\eta}^{-1}(x\lceil\eta))=x\lceil\eta\right.\right.$ by definition.

To prove that $H$ is projection-keeping, let $x_{0}, x_{1} \in X$. Assume that $\xi \in \mathbf{I S}_{\zeta}$ and $x_{0} \uparrow \xi=x_{1} \uparrow \xi$; we have to check that $H\left(x_{0}\right)\left\lceil\xi=H\left(x_{1}\right)\lceil\xi\right.$. Since $G$ is projection-keeping, it is enough to prove that the points

$$
z_{e}=G^{-1}\left(H\left(x_{e}\right)\right)=G_{\eta}^{-1}\left(x_{e}\lceil\eta) \cup F^{-1}\left(x_{e}\right)\lceil(\zeta \backslash \eta), \quad e=0,1,\right.
$$

satisfy $z_{0}\left\lceil\xi=z_{1}\left\lceil\xi\right.\right.$. Now $z_{e}\left\lceil\xi=G_{\xi^{\prime}}^{-1}\left(x_{e}\left\lceil\xi^{\prime}\right) \cup F_{\xi}^{-1}\left(x_{e}\lceil\xi)\left\lceil\xi^{\prime \prime}\right.\right.\right.\right.$, where $\xi^{\prime}=\xi \cap \eta$ and $\xi^{\prime \prime}=\xi \backslash \eta$, so that $z_{0}\left\lceil\xi=z_{1}\left\lceil\xi\right.\right.$ because $x_{0}\left\lceil\xi=x_{1}\lceil\xi\right.$ and both $F$ and $G$ are projection-keeping. The converse is similar.

Lemma 10. Suppose that $X \in \operatorname{Perf}_{\zeta}, \eta \in \mathbf{I S}_{\zeta}, Y \in \operatorname{Perf}_{\eta}$, and $Y \subseteq X\lceil\eta$. Then $Z=X \cap\left(Y \uparrow^{-1} \zeta\right)$ belongs to $\operatorname{Perf}_{\zeta}$.

Proof. Let $F: \mathcal{D}^{\zeta} \stackrel{\text { onto }}{\longrightarrow} X$ and $G: \mathcal{D}^{\eta} \stackrel{\text { onto }}{\longrightarrow} Y$ witness that $X \in \operatorname{Perf}_{\zeta}$ and $Y \in \operatorname{Perf}_{\eta}$ resp. Define a projection-keeping homeomorphism

$$
H(z)=F\left(F_{\eta}^{-1}(G(z\lceil\eta)) \cup(z\lceil(\zeta \backslash \eta)))\right.
$$

for all $z \in \mathcal{D}^{\zeta}$. We check that $H$ maps $\mathcal{D}^{\zeta}$ onto $Z$. Let $z \in \mathcal{D}^{\zeta}$. Then $H(z) \in$ $X$ by the choice of $F$. Moreover, $H(z)\left\lceil\eta=F_{\eta}\left(F_{\eta}^{-1}(G(z\lceil\eta)))=G(z\lceil\eta) \in Y\right.\right.$, so $H(z) \in Z$. Let conversely $z^{\prime} \in Z$, so that $z^{\prime}=F(x)$ for some $x \in \mathcal{D}^{\zeta}$. We define $z \in \mathcal{D}^{\zeta}$ by $z=G^{-1}\left(F_{\eta}(x\lceil\eta)) \cup(x \uparrow(\zeta \backslash \eta))\right.$. (To make sure that $G^{-1}$ is applicable note that $F_{\eta}\left(x\lceil\eta)=F(x)\left\lceil\eta=z^{\prime}\lceil\eta \in Z\lceil\eta=Y\right.\right.$.) Then by definition $H(z)=F(x)=z^{\prime}$.

We prove that $H$ is projection-keeping. Let $z_{0}, z_{1} \in \mathcal{D}^{\zeta}$ and $\xi \in \mathbf{I S}_{\zeta}$. Suppose that $z_{0}\left|\xi=z_{1}\right| \xi$, and prove $H\left(z_{0}\right)\left|\xi=H\left(z_{1}\right)\right| \xi$. Define $x_{e} \in \mathcal{D}^{\zeta}$ $(e=0,1)$ by $x_{e}=F_{\eta}^{-1}\left(G\left(z_{e}\lceil\eta)\right) \cup\left(z_{e}\lceil(\zeta \backslash \eta))\right.\right.$. Then, first, $H\left(z_{e}\right)=F\left(x_{e}\right)$, 
second, since both $F$ and $G$ are projection-keeping, we have $x_{0}\left\lceil\xi=x_{1}\lceil\xi\right.$ and then $F\left(x_{0}\right)\left|\xi=F\left(x_{1}\right)\right| \xi$, as required. The converse is proved analogously.

Lemma 11. Assume that $\zeta \subseteq \vartheta \in \mathbf{C P O}, X, Y \in \operatorname{Perf}_{\zeta}$, and $H$ is a projection-keeping homeomorphism $X$ onto $Y$. Then the sets $X^{\prime}=X \uparrow^{-1} \vartheta$ and $Y^{\prime}=Y \uparrow^{-1} \vartheta$ belong to $\operatorname{Perf}_{\vartheta}$, and the function $H^{\prime}$, defined on $X^{\prime}$ by $H^{\prime}\left(x^{\prime}\right) \uparrow(\vartheta \backslash \zeta)=x^{\prime} \uparrow(\vartheta \backslash \zeta)$ and $H^{\prime}\left(x^{\prime}\right) \uparrow \zeta=H\left(x^{\prime} \uparrow \zeta\right)$, is a projection-keeping homeomorphism $X^{\prime}$ onto $Y^{\prime}$.

Proof. If $X \in \operatorname{Perf}_{\zeta}$ is witnessed by a projection-keeping homeomorphism $F: \mathcal{D}^{\zeta} \stackrel{\text { onto }}{\longrightarrow} X$ then the homeomorphism $F^{\prime}$, defined on $\mathcal{D}^{\vartheta}$ by $F^{\prime}\left(x^{\prime}\right) \uparrow(\vartheta \backslash \zeta)=x^{\prime} \uparrow(\vartheta \backslash \zeta)$ and $F^{\prime}\left(x^{\prime}\right)\left\lceil\zeta=F\left(x^{\prime} \uparrow \zeta\right)\right.$ for all $x^{\prime} \in \mathcal{D}^{\vartheta}$, witnesses that $X^{\prime} \in \operatorname{Perf}_{\vartheta}$. The rest of the proof is equally simple.

2. Splitting technique. We shall exploit the construction of sets in $\operatorname{Perf}_{\zeta}$ as $X=\bigcap_{m \in \omega} \bigcup_{u \in 2^{m}} X_{u}$, where every $X_{u}$ belongs to Perf ${ }_{\zeta}$. This section introduces the technique.

First of all we specify requirements which imply an appropriate behaviour of the sets $X_{u} \in \operatorname{Perf}_{\zeta}$ with respect to projections. We need to determine, for any pair of finite binary sequences $u, v \in 2^{m}(m \in \omega)$, the largest initial segment $\xi=\zeta[u, v]$ of $\zeta$ such that the projections $X_{u}\lceil\xi$ and $X_{v}\lceil\xi$ have to be equal, to run the construction in a proper way.

Fix $\zeta \in \mathbf{C P O}$ and an arbitrary function $\phi: \omega \rightarrow \zeta$.

Define, for any pair of sequences $u, v \in 2^{m}(m \in \omega)$, an initial segment

$$
\begin{aligned}
\zeta_{\phi}[u, v] & =\bigcap_{l<m, u(l) \neq v(l)}[\geq \phi(l)] \\
& =\{j \in \zeta: \neg \exists l<m(u(l) \neq v(l) \& j \geq \phi(l))\} \in \mathbf{I S}_{\zeta} .
\end{aligned}
$$

Definition. A $\phi$-splitting system (or rather $(\phi\lceil m)$-splitting as the notion depends only on $\phi\left\lceil m\right.$ ) of order $m$ in $\operatorname{Perf}_{\zeta}$ is a family $\left\langle X_{u}: u \in 2^{m}\right\rangle$ of sets $X_{u} \in \operatorname{Perf}_{\zeta}$ such that

S-1. $X_{u}\left\lceil\zeta_{\phi}[u, v]=X_{v}\left\lceil\zeta_{\phi}[u, v]\right.\right.$ for all $u, v \in 2^{m}$.

S-2. If $i \in \zeta \backslash \zeta_{\phi}[u, v]$ then $\left.\left.X_{u}\right|_{\leq i} \cap X_{v}\right|_{\leq i}=\emptyset$ for all $u, v \in 2^{m}$.

A splitting system $\left\langle X_{u^{\prime}}: u^{\prime} \in 2^{m+1}\right\rangle$ is an expansion of a splitting system $\left\langle X_{u}: u \in 2^{m}\right\rangle$ iff $X_{u^{\wedge} e} \subseteq X_{u}$ for all $u \in 2^{m}$ and $e=0,1\left({ }^{5}\right)$.

We consider two ways how an existing splitting system can be transformed to another splitting system. One of them treats the case when we have to change one of the sets to a smaller set in $\operatorname{Perf} \zeta$, the other one expands to the next level.

$\left({ }^{5}\right)$ The characters $e, d$ will always denote the numbers 0 and 1. 
Lemma 12. Assume that $\left\langle X_{u}: u \in 2^{m}\right\rangle$ is a $\phi$-splitting system in $\operatorname{Perf}_{\zeta}, u_{0} \in 2^{m}$, and $X \in \operatorname{Perf}_{\zeta}, X \subseteq X_{u_{0}}$. Then the family of sets $X_{u}^{\prime}=X_{u} \cap\left(X\left\lceil\left.\zeta_{\phi}\left[u, u_{0}\right]\right|^{-1} \zeta\right)\left(u \in 2^{m}\right)\right.$ is again a $\phi$-splitting system. (Notice that $X_{u_{0}}^{\prime}=X$.)

Pr o of. Each set $X_{u}^{\prime}$ belongs to Perf $\mathrm{f}_{\zeta}$ by Lemmas 6 and 10 . We have to check only requirement S-1. Thus let $u, v \in 2^{m}$ and $\xi=\zeta_{\phi}[u, v]$. We prove that $X_{u}^{\prime}\left|\xi=X_{v}^{\prime}\right| \xi$. Define $\zeta_{u}=\zeta_{\phi}\left[u, u_{0}\right]$ and $\zeta_{v}=\zeta_{\phi}\left[v, u_{0}\right]$. Then

$$
X_{u}^{\prime} \uparrow \xi=\left(X_{u} \uparrow \xi\right) \cap\left(X \uparrow\left(\xi \cap \zeta_{u}\right) \uparrow^{-1} \xi\right), \quad X_{v}^{\prime} \uparrow \xi=\left(X_{v} \uparrow \xi\right) \cap\left(X \uparrow\left(\xi \cap \zeta_{v}\right) \uparrow^{-1} \xi\right)
$$

by Lemma 5 . Thus it remains to prove that $\xi \cap \zeta_{u}=\xi \cap \zeta_{v}$ (the "triangle" equality). Assume on the contrary that say $i \in \xi \cap \zeta_{u}$ but $i \notin \zeta_{v}$. Then $i \geq \phi(l)$ in $\zeta$ for some $l<m$ such that $v(l) \neq u_{0}(l)$. But then either $u(l) \neq u_{0}(l)$ and so $i \notin \zeta_{u}$, or $u(l) \neq v(l)$ and so $i \notin \xi$, a contradiction.

We are going to prove that each splitting system has an expansion. This requires defining first a special splitting construction.

Let $i \in \zeta$ and $X \in \operatorname{Perf}_{\zeta}$. A pair of sets $X_{0}, X_{1} \in \operatorname{Perf}_{\zeta}$ will be called an $i$-splitting of $X$ if $X_{0} \cup X_{1} \subseteq X, X_{0} \uparrow_{\geq i}=X_{1} \uparrow_{\geq i}$, and $X_{0} \uparrow_{\leq i} \cap X_{1} \uparrow_{\leq i}=\emptyset$. The splitting will be called complete if $X_{0} \cup X_{1}=X$; in this case we have $X_{0} \uparrow_{\searrow i}=X_{1} \uparrow_{\searrow i}=X \uparrow_{\searrow i}$.

Assertion. Let $i \in \zeta$. Every set $X \in \operatorname{Perf}_{\zeta}$ admits a complete $i$-splitting.

Proof. If $X=\mathcal{D}^{\zeta}$ then we define $X_{e}=\{x \in X: x(i)(0)=e\}$ for each $e=0,1$. Lemma 7 extends the result to the general case.

Lemma 14. Every $\phi$-splitting system $\left\langle X_{u}: u \in 2^{m}\right\rangle$ in $\operatorname{Perf}_{\zeta}$ can be expanded to a $\phi$-splitting system $\left\langle X_{u^{\prime}}: u^{\prime} \in 2^{m+1}\right\rangle$ in $\operatorname{Perf}_{\zeta}$.

Proof. Write $\zeta[u, v]$ instead of $\zeta_{\phi}[u, v]$ as $\phi$ is fixed. Let $i=\phi(m)$.

Let us consider, one by one in an arbitrary but fixed order, all sequences $u \in 2^{m}$. At each step $u$, we shall $i$-split $X_{u}$ in one of two different ways.

Case $A$. There does not exist $w \in 2^{m}$, considered earlier than $u$, such that $i \in \zeta[u, w]$. Let $X_{u^{\wedge} 0}, X_{u^{\wedge} 1}$ be any complete $i$-splitting of $X_{u}$.

Case $B$. Otherwise, let $w$ be the one considered first among all sequences $w$ of the mentioned type. Set $X_{u^{\wedge} e}=X_{u} \cap\left(\left.\left.X_{w^{\wedge} e}\right|_{<i}\right|^{-1} \zeta\right)$ for $e=0,1$.

We prove that $X_{u^{\wedge} 0}, X_{u^{\wedge} 1}$ is a complete $i$-splitting of $X_{u}$ in this case. First of all, $X_{u}\left\lceil\zeta[u, w]=X_{w}\left\lceil\zeta[u, w]\right.\right.$ by S-1; hence $\left.\left.X_{w \wedge e}\right|_{\leq i} \subseteq X_{w}\right|_{\leq i}=$ $\left.X_{u}\right|_{<i}$, so that the sets $X_{u^{\wedge} 0}$ and $X_{u^{\wedge} 1}$ belong to Perf $\mathcal{P}_{\zeta}$ by Lemmas 6 and 10 .

By the choice of $w$, we had Case A at step $w$. (Indeed, if otherwise $i \in \zeta\left[w, w^{\prime}\right]$ for some $w^{\prime} \in 2^{m}$ considered even earlier, then $i \in \zeta\left[u, w^{\prime}\right]$ by the "triangle" equality in the proof of Lemma 12, contrary to the choice of $w$.) Therefore for sure $X_{w^{\wedge} 0}, X_{w^{\wedge} 1}$ is a complete $i$-splitting of $X_{w}$. In particular, $X_{w^{\wedge} e} \Upsilon_{<i}=X_{w} \Upsilon_{<i}$. On the other hand, Lemma 5 implies $X_{u^{\wedge} e} \Upsilon_{\geq i}=X_{u} \Upsilon_{\geq i} \cap$ 
$\left(X_{w^{\wedge} e} \Gamma_{<i} \Gamma^{-1}[\not i]\right)$ for $e=0,1$, since $[\nsupseteq i] \cap[\leq i]=[<i]$ 一 so we get $X_{u^{\wedge} 0} \Upsilon_{\nsupseteq i}=$ $\left.X_{u \wedge 1}\right\rceil_{\geq i}$.

By definition, $\left.X_{u^{\wedge} e}\right|_{<i}=\left.X_{w^{\wedge} e}\right|_{<i}$ for $e=0,1$, so that $\left.X_{u^{\wedge} 0}\right|_{<i} \cap$ $\left.X_{u^{\wedge} 1}\right|_{\leq i}=\emptyset$ because $\bar{X}_{w^{\wedge} 0}, X_{w^{\wedge} 1}$ is a splitting of $X_{w}$. Finally, since $X_{w^{\wedge} 0}, X_{w^{\wedge} 1}$ is a complete $i$-splitting of $X_{w}$, and $\left.X_{w}\right|_{\leq i}=\left.X_{u}\right|_{\leq i}$, we have $X_{u^{\wedge} 0} \cup X_{u^{\wedge} 1}=X_{u}$, as required.

Thus $X_{u^{\wedge} 0}, X_{u^{\wedge} 1}$ is a complete $i$-splitting of $X_{u}$ for all $u \in 2^{m}$. It remains to prove that $\left\langle X_{u^{\prime}}: u^{\prime} \in 2^{m+1}\right\rangle$ is a splitting system.

To prove S-1 and S-2, let $u^{\prime}=u^{\wedge} d$ and $v^{\prime}=v^{\wedge} e$ belong to $2^{m+1}$; here $d, e \in\{0,1\}$. Define $\xi=\zeta[u, v], \xi^{\prime}=\zeta\left[u^{\prime}, v^{\prime}\right]$, and $Y=X_{u}\left|\xi=X_{v}\right| \xi$. We consider three cases.

Case 1: $i \notin \xi$. Then by definition $\xi=\xi^{\prime} \subseteq[\not i]$. We have $X_{u^{\prime}} \mid \xi=$ $X_{u} \mid \xi=Y$ because $X_{u^{\wedge} 0}, X_{u^{\wedge} 1}$ is a complete $i$-splitting of $X_{u}$. Similarly $X_{v^{\prime}} \uparrow \xi=Y$. This proves S-1 for the sets $X_{u^{\prime}}, X_{v^{\prime}}$, while S-2 is inherited from the pair $X_{u}, X_{v}$ because $\xi=\xi^{\prime}$ and $X_{u^{\prime}} \subseteq X_{u}, X_{v^{\prime}} \subseteq X_{v}$.

Case 2: $i \in \xi$ and $d=e$, say $d=e=0$. Then again $\xi=\xi^{\prime}$ by definition, so S-2 is clear, but $i \in \xi^{\prime}$. To prove S-1, let $w \in 2^{m}$ be the first (in the order fixed at the beginning of the proof) sequence in $2^{m}$ such that $i \in \zeta[u, w] \cup \zeta[v, w]$ (e.g. $w$ can be one of $u, v$ ). Then, since $i \in \xi=\zeta[u, v]$, we have $i \in \zeta[u, w] \cap \zeta[v, w]$ by the "triangle" equality. Finally, it follows from the construction (Case B) that

$$
X_{u^{\wedge} 0}\left\lceil\xi=\left(X_{u} \mid \xi\right) \cap\left(X_{w^{\wedge} 0} \Upsilon_{\leq i}\right\rceil^{-1} \xi\right), \quad X_{v^{\wedge} 0}\left\lceil\xi=\left(X_{v} \mid \xi\right) \cap\left(\left.X_{w^{\wedge} 0} \uparrow_{\leq i}\right|^{-1} \xi\right) .\right.
$$

However, $X_{u}\left|\xi=X_{v}\right| \xi=Y$; hence $X_{u^{\wedge} 0}\left|\xi^{\prime}=X_{v^{\wedge} 0}\right| \xi^{\prime}$ as $\xi^{\prime}=\xi$.

Case 3: $i \in \xi$ but $d \neq e$, say $d=0, e=1$. Now $\xi^{\prime}=\xi \cap[\nsupseteq i]$ is a proper initial segment of $\xi$. Let $w$ be introduced as in Case 2. Note that $\xi^{\prime} \cap[\leq i]=[<i]$, so $X_{u^{\wedge} 0}\left\lceil\xi^{\prime}=\left(X_{u} \mid \xi^{\prime}\right) \cap\left(\left.\left.X_{w^{\wedge} 0}\right|_{<i}\right|^{-1} \xi^{\prime}\right)\right.$ and $X_{v^{\wedge} 1} \mid \xi^{\prime}=$ $\left(X_{v} \mid \xi^{\prime}\right) \cap\left(\left.\left.X_{w^{\wedge} 1}\right|_{<i}\right|^{-1} \xi^{\prime}\right)$ by the construction and Lemma 5. However, we have $X_{w^{\wedge} 0} \Upsilon_{<i}=\left.X_{w^{\wedge} 1}\right|_{<i}$ because the pair $X_{w^{\wedge} 0}, X_{w^{\wedge} 1}$ is an $i$-splitting of $X_{w}$. Furthermore, $X_{u} \mid \xi^{\prime}=X_{v} \uparrow \xi^{\prime}=Y\left\lceil\xi^{\prime}\right.$ because $X_{u} \mid \xi=X_{v} \uparrow \xi=Y$. We conclude that $X_{u^{\wedge} 0}\left|\xi^{\prime}=X_{v^{\wedge} 1}\right| \xi^{\prime}$, as required.

Let us prove S-2 for some $i^{\prime} \in \zeta \backslash \xi^{\prime}$. If $i^{\prime} \notin \xi$ then already $\left.X_{u}\right|_{\leq i^{\prime}} \cap$ $\left.X_{v}\right|_{<i^{\prime}}=\emptyset$. If $i^{\prime} \in \xi \backslash \xi^{\prime}$ then $i^{\prime} \geq i$, so that it suffices to prove S-2 only for $i^{\prime}=i=\phi(m)$. To prove S-2 in this case, note that $\left.X_{u^{\wedge} 0}\right|_{\leq i}=\left.X_{w^{\wedge} 0}\right|_{\leq i}$ and $\left.X_{v^{\wedge} 1}\right|_{\leq i}=\left.X_{w^{\wedge} 1}\right|_{\leq i}$ by the construction. But $\left.\left.X_{w^{\wedge} 0}\right|_{\leq i} \cap X_{w^{\wedge} 1}\right|_{\leq i}=\emptyset$ as the pair $X_{w^{\wedge} 0}, X_{w^{\wedge} 1}$ is an $i$-splitting, so $\left.\left.X_{u^{\wedge} 0}\right|_{\leq i} \cap X_{v^{\wedge} 1}\right|_{\leq i}=\emptyset$.

To formulate the fusion lemma we need a couple more definitions.

Definition. An indexed family of sets $X_{u} \in \operatorname{Perf}_{\zeta}, u \in 2^{<\omega}$, is a $\phi$-fusion sequence in $\operatorname{Perf}_{\zeta}$ if for every $m \in \omega$ the subfamily $\left\langle X_{u}: u \in 2^{m}\right\rangle$ is a $\phi$-splitting system expanded by $\left\langle X_{u}: u \in 2^{m+1}\right\rangle$ to the next level, and 
S-3. For any $\varepsilon>0$ there exists $m \in \omega$ such that $\operatorname{diam} X_{u}<\varepsilon$ for all $u \in 2^{m}$. (A Polish metric on $\mathcal{D}^{\zeta}$ is assumed to be fixed.)

Definition. A function $\phi: \omega \rightarrow \zeta$ is $\zeta$-complete iff it takes each value $i \in \zeta$ infinitely many times.

Theorem 15 (Fusion lemma). Let $\phi$ be a $\zeta$-complete function. Suppose that $\left\langle X_{u}: u \in 2^{<\omega}\right\rangle$ is a $\phi$-fusion sequence in $\operatorname{Perf}_{\zeta}$. Then the set $X=$ $\bigcap_{m \in \omega} \bigcup_{u \in 2^{m}} X_{u}$ belongs to $\operatorname{Perf}_{\zeta}$.

Proof. The idea of the proof is to obtain a parallel presentation of the set $D=\mathcal{D}^{\zeta}$ as the "limit" of a $\phi$-fusion sequence, and associate the points in $D$ and $X$ which are generated by one and the same branch in $2^{<\omega}$. So first of all we have to define a fusion sequence of sets $D_{u} \in \operatorname{Perf}_{\zeta}$ such that $\mathcal{D}^{\zeta}=D=\bigcap_{m \in \omega} \bigcup_{u \in 2^{m}} D_{u}$.

Lemma 14 cannot be used because of problems with S-3. We rather maintain a direct construction. For $m \in \omega$, we put $\zeta_{m}=\{\phi(l): l<m\}$. Let $i \in \zeta_{m}$, and $\{l<m: \phi(l)=i\}=\left\{l_{0}^{i}, \ldots, l_{k(i)-1}^{i}\right\}$, in the increasing order. If $u \in 2^{m}$ then we define $u_{i} \in 2^{k(i)}$ by $u_{i}(k)=u\left(l_{k}^{i}\right)$ for all $k<k(i)$, and put $D_{u}=\left\{y \in D=\mathcal{D}^{\zeta}: \forall i \in \zeta_{m}\left(u_{i} \subset y(i)\right)\right\}$, so that $D_{u}$ is a basic clopen set in $\mathcal{D}^{\zeta}$. (Note that $y(i) \in \mathcal{D}$ whenever $y \in \mathcal{D}^{\zeta}$ and $i \in \zeta$.) One easily sees that the sets $D_{u}$ form a $\phi$-fusion sequence (S-3 follows from the $\zeta$-completeness of $\phi$ ) and $\bigcup_{u \in 2^{m}} D_{u}=\mathcal{D}^{\zeta}$ for all $m$.

We observe that for each $a \in 2^{\omega}=\mathcal{D}$ the intersections $\bigcap_{m} X_{a \uparrow m}$ and $\bigcap_{m} D_{a \uparrow m}$ contain single points, say $x_{a} \in X$ and $d_{a} \in D$ respectively, by $\mathrm{S}-3$, and the mappings $a \mapsto x_{a}$ and $a \mapsto d_{a}$ are continuous. Let us define $\zeta_{\phi}[a, b]=\bigcap_{m \in \omega} \zeta_{\phi}\left[a\left\lceil m, b\lceil m]\right.\right.$. In particular $\zeta_{\phi}[a, b]=\zeta$ iff $a=b$. It follows from the requirements $\mathrm{S}-1, \mathrm{~S}-2$, and $\mathrm{S}-3$ that

$$
\left\{\begin{array}{l}
x_{a}\left\lceil\zeta_{\phi}[a, b]=x_{b}\left\lceil\zeta_{\phi}[a, b]\right.\right. \\
d_{a}\left\lceil\zeta_{\phi}[a, b]=d_{b}\left\lceil\zeta_{\phi}[a, b]\right.\right. \\
\left.x_{a}\right|_{\leq i} \neq\left. x_{b}\right|_{\leq i} \text { and }\left.d_{a}\right|_{\leq i} \neq\left. d_{b}\right|_{\leq i} \quad \text { whenever } i \notin \zeta_{\phi}[a, b] .
\end{array}\right.
$$

This allows us to define a homeomorphism $H: D=\mathcal{D}^{\zeta} \stackrel{\text { onto }}{\longrightarrow} X$ by $H\left(d_{a}\right)=$ $x_{a}$ for all $a \in 2^{\omega}$. To see that $H$ is projection-keeping let $\xi \in \mathbf{I S}_{\zeta}$ and, for instance, $d_{a}, d_{b} \in \mathcal{D}^{\zeta}$ and $d_{a}\left\lceil\xi=d_{b}\left\lceil\xi\right.\right.$. Then $\xi \subseteq \zeta_{\phi}[a, b]$ by the second line in $(*)$, so we get $x_{a}\left|\xi=x_{b}\right| \xi$ by the first line, as required.

Corollary 16. Suppose that $X \in \operatorname{Perf}_{\zeta}$, and $C_{m} \subseteq \mathcal{D}^{\zeta}$ is closed for each $m \in \omega$. There exists $Y \in \operatorname{Perf}_{\zeta}, Y \subseteq X$, such that $C_{m} \cap Y$ is clopen in $Y$ for every $m$.

Proof. It follows from Lemma 8 that for any $m$ and any $X^{\prime} \in \operatorname{Perf}_{\zeta}$ there exists $Y^{\prime} \in \operatorname{Perf}_{\zeta}, Y^{\prime} \subseteq X^{\prime}$, such that either $Y^{\prime} \subseteq C_{m}$ or $Y^{\prime} \cap C_{m}=\emptyset$. Therefore we can define, using Lemmas 12 and 14, a fusion sequence $\left\langle X_{u}\right.$ : 
$\left.u \in 2^{<\omega}\right\rangle$ of sets $X_{u} \in \operatorname{Perf}_{\zeta}$ such that $X_{\Lambda}=X$ and either $X_{u} \subseteq C_{m}$ or $X_{u} \cap C_{m}=\emptyset$ whenever $u \in 2^{m}$ - for all $m \in \omega$. The set $Y=\bigcap_{m \in \omega} \bigcup_{u \in 2^{m}} X_{u}$ is as required.

Corollary 17. Assume that $X \in \operatorname{Perf}_{\zeta}$, and $B \subseteq \mathcal{D}^{\zeta}$ is a set of a finite Borel rank. There exists $Y \in \operatorname{Perf}_{\zeta}, Y \subseteq X$, such that either $Y \subseteq B$ or $Y \cap B=\emptyset$.

Pr o of $\left({ }^{6}\right)$. Let $B$ be defined by a finite level Borel scheme (countable unions plus countable intersections) from closed sets $C_{m}, m \in \omega$. The preceding corollary shows that there exists $X^{\prime} \in \operatorname{Perf}_{\zeta}, X^{\prime} \subseteq X$, such that every $X^{\prime} \cap C_{m}$ is clopen in $X^{\prime}$. Thus the Borel rank can be reduced. When one finally achieves the level of closed or open sets, Lemma 8 is applied.

3. Reducibility of continuous functions. This section studies the behaviour of continuous functions defined on sets in $\operatorname{Perf}_{\zeta}, \zeta \in \mathbf{C P O}$, from the point of view of a certain reducibility.

Definition. For each set $\zeta$, $\operatorname{Cont}_{\zeta}$ will denote the set of all continuous functions $F: \mathcal{D}^{\zeta} \rightarrow$ reals. (As usual, reals $=\mathcal{N}=\omega^{\omega}$.)

Let $F \in \operatorname{Cont}_{\zeta}, \xi \subseteq \zeta, X \subseteq \mathcal{D}^{\zeta}, i \in \zeta$. Then $F$ reduces to $\xi$ on $X$ iff $x \nmid \xi=y\lceil\xi \Rightarrow F(x)=F(y)$ for all $x, y \in X$; and $F$ captures $i$ on $X$ iff $F(x)=F(y) \Rightarrow x(i)=y(i)$ for all $x, y \in X$.

Remark. It follows from the compactness of the spaces $\mathcal{D}^{\zeta}$ that if $X$ is closed then in the first case there is a function $F^{\prime} \in \operatorname{Cont}_{\xi}$ such that $F(x)=F^{\prime}(x \mid \xi)$ for all $x \in X$, while in the second case there is a continuous function $H$ : reals $\rightarrow \mathcal{D}$ such that $x(i)=H(F(x))$ for all $x \in X$.

We begin with two technical lemmas on reducibility. Then an important theorem will be proved; different items of the theorem will be transformed to properties of constructibility of reals in the generic extensions.

Lemma 18. Let $\xi, \eta \in \mathbf{I S}_{\zeta}$. If $F$ reduces to both $\xi$ and $\eta$ on a set $X \in$ $\operatorname{Perf}_{\zeta}$ then $F$ reduces to $\vartheta=\xi \cap \eta$ on $X$.

Proof. Let $x, y \in X$ and $x\lceil\vartheta=y\lceil\vartheta$. By Proposition 3 (P-3) there is $z \in X$ such that $z\lceil\xi=x\lceil\xi$ and $z\lceil\eta=y\lceil\eta$. Now $F(x)=F(z)=F(y)$.

Lemma 19. Suppose that $\xi \in \mathbf{I S}_{\zeta}$, the sets $X_{1}$ and $X_{2}$ belong to $\operatorname{Perf}_{\zeta}$, and $X_{1}\left|\xi=X_{2}\right| \xi$. Then either $F$ reduces to $\xi$ on $X_{1} \cup X_{2}$-and then $F^{\prime \prime} X_{1}=F " X_{2}$, -or there are sets $X_{1}^{\prime}, X_{2}^{\prime} \in \operatorname{Perf}_{\zeta}, X_{1}^{\prime} \subseteq X_{1}$ and $X_{2}^{\prime} \subseteq X_{2}$ such that still $X_{1}^{\prime} \uparrow \xi=X_{2}^{\prime} \mid \xi$, but $F^{\prime \prime} X_{1}^{\prime} \cap F^{\prime \prime} X_{2}^{\prime}=\emptyset$.

(We recall that $F " X$ is the image of $X$ via $F$.)

$\left({ }^{6}\right)$ In fact, this is true for all Borel sets $B$ but needs a more elaborate reasoning. 
Proof. We assume that the function $F$ does not reduce to $\xi$ on the set $X_{1} \cup X_{2}$, and prove the "or" alternative. By the assumption, there are points $x_{1}, x_{2} \in X_{1} \cup X_{2}$ satisfying $x_{1} \uparrow \xi=x_{2} \uparrow \xi$ and $F\left(x_{1}\right) \neq F\left(x_{2}\right)$. It may be supposed that $x_{1} \in X_{1}$ and $x_{2} \in X_{2}$, because $X_{1} \mid \xi=X_{2} \uparrow \xi$. By the continuity of $F$ there exist clopen neighbourhoods $U_{1}$ and $U_{2}$ of resp. $x_{1}$ and $x_{2}$ such that $F^{\prime \prime} U_{1} \cap F^{\prime \prime} U_{2}=\emptyset$. Now Lemma 8 provides a set $X_{1}^{\prime \prime} \in \operatorname{Perf}_{\zeta}$, $X_{1}^{\prime \prime} \subseteq X_{1} \cap U_{1}$, containing $x_{1}$.

The set $X_{2}^{\prime \prime}=X_{2} \cap\left(X_{1}^{\prime \prime}|\xi|^{-1} \zeta\right)$ belongs to $\operatorname{Perf} f_{\zeta}$ by Lemma 10, and contains $x_{2}$ since $x_{1}\left|\xi=x_{2}\right| \xi$. By Lemma 8 there is a set $X_{2}^{\prime} \in \operatorname{Perf}_{\zeta}$ satisfying $X_{2}^{\prime} \subseteq X_{2}^{\prime \prime} \cap U_{2}$. Define $X_{1}^{\prime}=X_{1}^{\prime \prime} \cap\left(X_{2}^{\prime}\left\lceil\xi \uparrow^{-1} \zeta\right)\right.$.

Theorem 20. Assume that $X \in \operatorname{Perf}_{\zeta}, \xi \in \mathbf{I S}_{\zeta}$ and $F \in$ Cont $_{\zeta}$. Then

(1) If $i, j \in \zeta$ and $i<j$ then there exists $Y \in \operatorname{Perf}_{\zeta}, Y \subseteq X$, such that the co-ordinate function $C_{j}$, defined on $\mathcal{D}^{\zeta}$ by $C_{j}(x)=x(j)$, captures $i$ on $Y$.

(2) If $i \in \zeta \backslash \xi$ and $F$ reduces to $\xi$ on $X$ then $F$ does not capture $i$ on $X$.

(3) If for each set $X^{\prime} \in \operatorname{Perf}_{\zeta}, X^{\prime} \subseteq X$, and each $i \in \xi$ there is a set $X^{\prime \prime} \in \operatorname{Perf}_{\zeta}, X^{\prime \prime} \subseteq X^{\prime}$, such that $F$ captures $i$ on $X^{\prime \prime}$, then there exists $Y \in \operatorname{Perf}_{\zeta}, Y \subseteq X$, such that $F$ captures every $i \in \xi$ on $Y$.

(4) If $i \in \zeta$, then there exists $Y \in \operatorname{Perf}_{\zeta}, Y \subseteq X$, such that either $F$ reduces to $[\nsupseteq i]$ on $Y$, or $F$ captures $i$ on $Y$.

(5) There exists a set $Y \in \operatorname{Perf}_{\zeta}, Y \subseteq X$, such that either $F$ reduces to $\xi$ on $Y$, or $F$ captures some $i \in \zeta \backslash \xi$ on $Y$.

$((4)$ is an easy corollary of (1) and (5), for take $\xi=[\nsupseteq i]$. However, we need it to be proved separately because it is used in the proof of (5).)

Proof. (2) Suppose that $F$ reduces to $\xi$ on $X$ and, on the contrary, $F$ does capture some $i \in \zeta \backslash \xi$ on $X$. Then the coordinate function $C_{i}(x)=$ $x(i)$ itself reduces to $\xi$ on $X$. Since $i$ does not belong to $\xi$, and on the other hand $C_{i}$ obviously reduces to $[\leq i]$, we conclude that $C_{i}$ reduces to $[<i]$ on $X$ by Lemma 18 . But this clearly contradicts property P-1 of $X$ (see Proposition 4).

(3) and (4) are carried out by a common construction. We define a set $Y \in \operatorname{Perf}_{\zeta}, Y \subseteq X$, which satisfies the "or" requirement of (4) unless an intermediate set in the construction satisfies the "either" requirement.

Fix a $\zeta$-complete function $\phi$ and define the initial segments $\zeta[u, v]=$ $\zeta_{\phi}[u, v]$ (as in Section 2) for every pair of finite sequences $u, v \in 2^{<\omega}$ of equal length. The notions of splitting system and fusion sequence are understood in the sense of $\phi$.

We define a fusion sequence $\left\langle X_{u}: u \in 2^{<\omega}\right\rangle$ satisfying $X_{\Lambda}=X$ and the property 
( ) If $m \in \omega$ and $u, v \in 2^{m}$ then either (i) $F$ reduces to $\zeta[u, v]$ on the set $X_{u} \cup X_{v}$, or (ii) $F " X_{u} \cap F^{\prime \prime} X_{v}=\emptyset$.

First we put $X_{\Lambda}=X$, as indicated.

Assume that sets $X_{u}\left(u \in 2^{m-1}\right)$ have been defined for some $m>0$. We use Lemma 14 to get a splitting system $\left\langle Z_{u}: u \in 2^{m}\right\rangle$ which expands the splitting system $\left\langle X_{u}: u \in 2^{m-1}\right\rangle$ already obtained to the level $m$. We can suppose that $\operatorname{diam} Z_{u} \leq m^{-1}$ for all $u \in 2^{m}$. (Otherwise apply Lemmas 8 and 12 consecutively $2^{m}$ times to shrink the sets.) We need this property to satisfy requirement S-3.

We now consider consecutively all pairs $u, v \in 2^{m}$. For every such pair we first apply Lemma 19, getting sets $S_{u}, S_{v} \in \operatorname{Perf}_{\zeta}$ such that $S_{u} \subseteq Z_{u}$, $S_{v} \subseteq Z_{v}, S_{u}\left\lceil\zeta[u, v]=S_{v}\lceil\zeta[u, v]\right.$, and either the function $F$ reduces to $\zeta[u, v]$ on $S_{u} \cup S_{v}$ or $F " S_{u} \cap F " S_{v}=\emptyset$.

We set $S_{w}^{\prime}=Z_{w} \cap\left(S_{u}\left\lceil\zeta[w, u] \uparrow^{-1} \zeta\right)\right.$ for all $w \in 2^{m} ;\left\langle S_{w}^{\prime}: w \in 2^{m}\right\rangle$ is a splitting system by Lemma 12 . Note that $S_{v} \subseteq S_{v}^{\prime}$ since $S_{u}\lceil\zeta[u, v]=$ $S_{v}\left\lceil\zeta[u, v]\right.$. We repeat the operation: putting $Z_{w}^{\prime}=S_{w}^{\prime} \cap\left(S_{v}\left\lceil\zeta[w, v] \uparrow^{-1} \zeta\right)\right.$ for all $w \in 2^{m}$, we obtain a new splitting system of sets $Z_{w}^{\prime} \subseteq S_{w}^{\prime}(w \in$ $2^{m+1}$ ) such that $Z_{u}^{\prime}=S_{u}$ and $Z_{v}^{\prime}=S_{v}$. This ends the consideration of the particular pair of $u, v \in 2^{m}$, and one comes to the next pair.

Let $X_{u} \subseteq Z_{u}\left(u \in 2^{m}\right)$ be the sets obtained after $2^{m+1}$ steps of this construction (the number of pairs $u, v$ to consider). One easily verifies that this is a splitting system in $\operatorname{Perf}_{\zeta}$ satisfying $(\ddagger)$ for the given $m$.

After the construction is accomplished for all $m$, we obtain a fusion sequence of sets $X_{u}\left(u \in 2^{<\omega}\right)$ satisfying $(\ddagger)$. The set $Y=\bigcap_{m} \bigcup_{u \in 2^{m}} X_{u}$ belongs to $\operatorname{Perf}_{\zeta}$ by Theorem 15.

(4) Suppose that we have case (i) in ( $\ddagger)$ for some pair $u, v \in 2^{m}(m \in \omega)$ such that $i \notin \zeta[u, v]$. Then in particular $F$ reduces to $\zeta[u, v]$ 一 then to $[¥ i]$ on $X_{u}$, so $X_{u}$ is a set of the "either" type.

Assume that (ii) in ( $\ddagger$ ) holds for all pairs $u, v \in 2^{m}(m \in \omega)$ such that $i \notin \zeta[u, v]$. We prove that then $F$ captures $i$ on the set $Y=\bigcap_{m} \bigcup_{u \in 2^{m}} X_{u}$. Let $x, y \in Y$. Suppose that $F(x)=F(y)$ and prove $x(i)=y(i)$.

Note that $x=x_{a}$ and $y=x_{b}$ for some $a, b \in 2^{\omega}$, i.e. $\{x\}=\bigcap_{m \in \omega} X_{a \uparrow m}$ and $\{y\}=\bigcap_{m \in \omega} X_{b \backslash m}$; see the proof of Theorem 15. Set $\zeta[a, b]=$ $\bigcap_{m} \zeta[a\lceil m, b\lceil m]$; then $x\lceil\zeta[a, b]=y\lceil\zeta[a, b]$ (see $(*)$ in the proof of Theorem 15), so it suffices to check $i \in \zeta[a\lceil m, b\lceil m]$ for all $m$.

Let $u=a\lceil m$ and $v=b\lceil m$. Suppose on the contrary that $i \notin \zeta[u, v]$. Then we have case (ii) in ( $\ddagger$ ) for $u, v$ by the assumption above - a contradiction since $F(x)=F(y)$.

(3) The set $Y$ proves this item, too. Suppose that $x, y \in Y$ satisfy $F(x)=$ $F(y)$; we show that $x\left\lceil\xi=y \mid \xi\right.$. As above, $x=x_{a}$ and $y=x_{b}$ for some $a, b \in 2^{\omega}$. It suffices to check that $\xi \subseteq \zeta[a\lceil m, b\lceil m]$ for all $m$. 
Let $i \in \xi$ and $m \in \omega$. Then we have case (ii) of ( $\ddagger$ ) for the pair $u=a\lceil m$, $v=b\lceil m$ (because $F(x)=F(y)$ ), so that in particular $F$ reduces to $\zeta[u, v]$ on $X_{u}$. By the assumption of (3), $F$ captures $i$ on some $Z \in \operatorname{Perf}_{\zeta}, Z \subseteq X_{u}$. But then $i \in \zeta[u, v]$ by $(2)$.

(1) Otherwise, by (4) the coordinate function $C_{j}$ would reduce to $\xi=[\nsupseteq i]$ on some $Y \in \operatorname{Perf}_{\zeta}, Y \subseteq X$, a contradiction with (2).

(5) Assume that a set $Y \in \operatorname{Perf}_{\zeta}$ of the "or" type of (5) does not exist. Then by (4), if $i \in \zeta \backslash \xi$ then every set $Y \in \operatorname{Perf}_{\zeta}, Y \subseteq X$, contains a subset $Z \in \operatorname{Perf}_{\zeta}$ such that $F$ reduces to $[\not i]$ on $Z$. Arguing as above, we obtain a fusion sequence $\left\langle X_{u}: u \in 2^{<\omega}\right\rangle$ such that $X_{\Lambda} \subseteq X$ and $F$ reduces to $[\nsupseteq \phi(m)]$ on $X_{u}$ whenever $u \in 2^{m}$ and $\phi(m) \notin \xi$. Then $Y=\bigcap_{m} \bigcup_{u \in 2^{m}} X_{u} \in \operatorname{Perf}_{\zeta}$.

We prove that $Y$ is a set of the "either" type, i.e. $F$ reduces to $\xi$ on $Y$.

Let us define, for every $m \in \omega$, an initial segment $\zeta_{m} \subseteq \zeta$ by

$$
\zeta_{m}=\bigcap_{l<m, \phi(l) \notin \xi}[\geq \phi(l)]=\{j \in \zeta: \neg \exists l<m(j \geq \phi(l) \notin \xi)\} .
$$

Then $\xi \subseteq \zeta_{m}$ for all $m$, and $\zeta_{m} \subseteq \zeta[u, v]$ whenever $u, v \in 2^{m}$ satisfy $\xi \subseteq$ $\zeta[u, v]$.

Assertion 21. For any $m, F$ reduces to $\zeta_{m}$ on $X_{m}=\bigcup_{u \in 2^{m}} X_{u}$.

Proof of the assertion (by induction on $m$ ). The case $m=0$ is trivial: $\zeta_{0}=\zeta$ by definition. Let us carry out the step from $m$ to $m+1$. Let $i=\phi(m)$. If $i \in \xi$ then $\zeta_{m+1}=\zeta_{m}$ and the statement is obvious. Therefore one can assume that $i=\phi(m) \notin \xi$. Then $F$ is reducible to [ $¥ i]$ on each set $X_{u^{\prime}}\left(u^{\prime} \in 2^{m}\right)$ by the construction of the fusion sequence.

Suppose that $u, v \in 2^{m+1}$, and points $x \in X_{u}$ and $y \in X_{v}$ satisfy the equality $x\left\lceil\zeta_{m+1}=y\left\lceil\zeta_{m+1}\right.\right.$, and prove $F(x)=F(y)$. We put $u^{\prime}=u\lceil m$ and $v^{\prime}=v\left\lceil m\right.$; then $u^{\prime}, v^{\prime} \in 2^{m}$.

We have $\zeta_{m+1} \subseteq \zeta[u, v]$ (otherwise $X_{u} \mid \zeta_{m+1} \cap X_{v} \uparrow \zeta_{m+1}=\emptyset$ by S-2, but $x \uparrow \zeta_{m+1}=y\left\lceil\zeta_{m+1}\right)$, therefore $\xi \subseteq \zeta[u, v]$ because every set $\zeta_{n}$ includes $\xi$. This implies $\xi \subseteq \zeta\left[u^{\prime}, v^{\prime}\right]$. It follows (see above) that $\zeta_{m} \subseteq \zeta\left[u^{\prime}, v^{\prime}\right]$. Therefore $X_{u^{\prime}} \uparrow \zeta_{m}=X_{v^{\prime}} \uparrow \zeta_{m}$ by S-1, so $y \uparrow \zeta_{m} \in X_{u^{\prime}} \uparrow \zeta_{m}$. We choose some $x^{\prime} \in X_{u^{\prime}}$ satisfying $x^{\prime} \uparrow \zeta_{m}=y \uparrow \zeta_{m}$. Then $F\left(x^{\prime}\right)=F(y)$ by the induction hypothesis, so it remains to verify that $F(x)=F\left(x^{\prime}\right)$.

Notice that $x$ and $x^{\prime}$ belong to $X_{u^{\prime}}$ and $x \backslash \zeta_{m+1}=x^{\prime} \mid \zeta_{m+1}$ by the choice of $x^{\prime}$. Thus it suffices to prove that $F$ reduces to $\zeta_{m+1}$ on $X_{u^{\prime}}$. We observe that, since $i=\phi(m) \notin \xi, F$ reduces to $[\nsupseteq i]$ on $X_{u^{\prime}}$ (see above). Moreover, $F$ reduces to $\zeta_{m}$ on $X_{u^{\prime}}$ by the induction hypothesis. Therefore $F$ reduces to $[\nsupseteq i] \cap \zeta_{m}$ on $X_{u^{\prime}}$ by Lemma 18. Finally, we have $\zeta_{m+1}=[\not i] \cap \zeta_{m}$ by definition.

We end the proof of (5) of Theorem 20. 
It follows from the assertion that $F$ reduces to every $\zeta_{m}$ on $Y$. This allows us to conclude that $F$ also reduces to $\xi$ on $Y$. (Indeed, assume on the contrary that $x, y \in Y$ and $x\lceil\xi=y \mid \xi$ but $F(x) \neq F(y)$. By the continuity of $F$ there exist $m \in \omega$ and $u, v \in 2^{m}$ such that $x \in X_{u}, y \in X_{v}$, and $F^{\prime \prime} X_{u} \cap F " X_{v}=\emptyset$. On the other hand, $\left(X_{u} \mid \xi\right) \cap\left(X_{v} \mid \xi\right) \neq \emptyset$, hence $\xi \subseteq \zeta[u, v]$ by S-2. This implies $\xi \subseteq \zeta_{m} \subseteq \zeta[u, v]$, as above. Therefore $F$ reduces to $\zeta[u, v]$ on $Y$, contradicting the equality $F^{\prime \prime} X_{u} \cap F^{\prime \prime} X_{v}=\emptyset$ since $X_{u} \uparrow \zeta[u, v]=X_{v} \uparrow \zeta[u, v]$ by $\mathrm{S}-1$.)

4. "Ill" founded iterated Sacks extensions. This section introduces generic models obtained by different sets $\operatorname{Perf}_{\zeta}$ as forcing notions. This will be detailed below towards particular applications.

Let $\mathfrak{M}$ be a countable transitive model of $\mathbf{Z F C}$, the ground model.

Fix a partially ordered set $\mathbf{I} \in \mathfrak{M}$ (generally speaking, uncountable in $\mathfrak{M})$ - the intended "length" of the planned Sacks iteration.

We define $\boldsymbol{\Xi}=\mathbf{C P O}^{\mathfrak{M}}(\mathbf{I}) \in \mathfrak{M}$ to be the collection of all finite and $\mathfrak{M}$-countable sets $\xi \in \mathfrak{M}, \xi \subseteq \mathbf{I}\left({ }^{7}\right)$, therefore $\boldsymbol{\Xi} \subseteq \mathbf{C P O}$ in $\mathfrak{M}$.

For any $\zeta \in \boldsymbol{\Xi}$, let $\mathbb{P}_{\zeta}=\left(\operatorname{Perf}_{\zeta}\right)^{\mathfrak{M}}$. The set $\mathbb{P}=\mathbb{P}_{\mathbf{I}}=\bigcup_{\zeta \in \boldsymbol{\Xi}} \mathbb{P}_{\zeta}$ will be the forcing notion. To define the order, we first put $\|X\|=\zeta$ whenever $X \in \mathbb{P}_{\zeta}$. Now we set $X \leq Y$ (i.e. $X$ is stronger than $Y$ ) iff $\zeta=\|Y\| \subseteq\|X\|$ and $X\lceil\zeta \subseteq Y$.

Notice that every set in $\mathbb{P}_{\zeta}$ is then a countable subset of $\mathcal{D}^{\zeta}$ in the universe. However, we can transform it to a perfect set in the universe by the closure operation: the topological closure $X^{\#}$ of a set $X \in \mathbb{P}_{\zeta}$ in $\mathcal{D}^{\zeta}$ belongs to $\operatorname{Perf}_{\zeta}$ from the point of view of the universe.

Let $G \subseteq \mathbb{P}$ be a $\mathbb{P}$-generic ultrafilter over $\mathfrak{M}$.

It easily follows from Lemma 8 that there exists a unique indexed set $\mathbf{x}=\left\langle\mathbf{a}_{i}: i \in \mathbf{I}\right\rangle$, all $\mathbf{a}_{i}=\mathbf{x}(i)$ being elements of $\mathcal{D}$, such that $\mathbf{x}\left\lceil\xi \in X^{\#}\right.$ whenever $X \in G$ and $\|X\|=\xi \in \mathbf{\Xi}$. Then $\mathfrak{M}[G]=\mathfrak{M}\left[\left\langle\mathbf{a}_{i}: i \in \mathbf{I}\right\rangle\right]=\mathfrak{M}[\mathbf{x}]$ is the generic extension of $\mathfrak{M}$.

In this section, we prove a "restriction" theorem for the forcing, a cardinal preservation theorem for the extension $\mathfrak{N}=\mathfrak{M}[G]$, and an important technical theorem which will allow us to study reals in $\mathfrak{N}$ using continuous functions in the ground model $\mathfrak{M}$. The next section will contain a more detailed study of reals in the extension. In particular, we shall prove that $\mathfrak{N}$ is in fact an iterated Sacks extension of $\mathfrak{M}$.

Restriction theorem. Suppose that $J \in \mathfrak{M}$ is an initial segment of $\mathbf{I}$. It often happens that in similar cases sentences relativized to $\mathfrak{M}[\mathbf{x}\lceil J]$ are

$\left({ }^{7}\right)$ If all initial segments of $\mathbf{I}$ with perhaps the exception of $\mathbf{I}$ itself are countable in $\mathfrak{M}$, it would be technically easier to define $\boldsymbol{\Xi}$ to be the set of all $\mathfrak{M}$-countable initial segments of $\mathbf{I}$ in $\mathfrak{M}$. 
decided by forcing conditions $X$ satisfying $\|X\| \subseteq J$. Thanks to projectionkeeping automorphisms, we can prove even more for the forcing $\mathbb{P}$. We start with a useful lemma, and then prove the restriction theorem.

Lemma 22. Suppose that $\eta, \xi \in \boldsymbol{\Xi}$ and $\forall j \in \eta \exists i \in \xi(j \leq i)$. Then $\mathbf{x}\lceil\eta \in \mathfrak{M}[\mathbf{x}\lceil\xi]$.

Pro of. Let the opposite be forced by some $X \in \mathbb{P}$. We may assume, by Lemma 11, that $\xi \cup \eta \subseteq \zeta=\|X\|$ and $\eta$ is an initial segment of $\zeta$.

We argue in $\mathfrak{M}$. So $X \in \operatorname{Perf}_{\zeta}$. Let $F$ be a continuous 1-1 map $\mathcal{D}^{\xi} \rightarrow \mathcal{D}$. Let $F^{\prime}(x)=F(x \mid \xi)$ for all $x \in \mathcal{D}^{\zeta}$. It follows from Theorem $20(1,3)$ that there exists $Y \in \operatorname{Perf}_{\zeta}, Y \subseteq X$, such that $F^{\prime}$ captures every $j \in \eta$ on $Y$, so we have a continuous map $H: Y\left\lceil\xi \rightarrow \mathcal{D}^{\eta}\right.$ such that $x\lceil\eta=H(x \mid \xi)$ for all $x \in X$.

But, since $Y$ obviously forces that $\mathbf{x} \backslash \zeta \in Y^{\#}$, we conclude, by the Shoenfield absoluteness theorem, that $Y$ forces $\mathbf{x}\left\lceil\eta=H^{\#}(\mathbf{x}\rceil \xi\right)$, where $H^{\#}$ is the topological closure of $H$ as a subset of $\mathcal{D}^{\xi} \times \mathcal{D}^{\eta}$ in a generic extension. Thus $Y$ forces $\mathbf{x}\lceil\eta \in \mathfrak{M}[\mathbf{x}\lceil\xi]$, a contradiction.

THEOREM 23. Suppose that $J$ is an initial segment of $\mathbf{I}, \Phi$ a sentence containing only $\mathbf{x} \mid J$ and sets in $\mathfrak{M}$ as parameters. Assume that $\tau \in \boldsymbol{\Xi}$, $\tau^{\prime}=\tau \cap J$, and a condition $Z \in \mathbb{P}_{\tau}$ forces $\Phi$. Then the weaker condition $Z^{\prime}=Z\left\lceil\tau^{\prime}\right.$ forces $\Phi$ as well. The result remains true if in addition $\Phi$ contains, as parameters, several (names of) sets of the form $\mathbf{x}\lceil\xi$, where $\xi \in \boldsymbol{\Xi}$ but not necessarily $\xi \subseteq J$, which enter the formula only through the expressions $\mathfrak{M}\left[\mathbf{x}\lceil\xi]\left({ }^{8}\right)\right.$.

Proof. Assume that this is not the case. We assert that there exist: $\zeta \in \boldsymbol{\Xi}$ and a pair of conditions $X, Y \in \mathbb{P}_{\zeta}$ such that $X\left\lceil\zeta^{\prime}=Y\left\lceil\zeta^{\prime}\right.\right.$, where $\zeta^{\prime}=\zeta \cap J, X$ forces $\Phi$, but $Y$ forces $\neg \Phi$.

(Indeed, let us argue in $\mathfrak{M}$. There exists a condition $Y$, stronger than $Z^{\prime}$, which forces $\neg \Phi$. We may assume, by Lemma 11, that $\tau \subseteq \zeta=\|Y\|$. Let $X^{\prime}=Z \uparrow^{-1} \zeta$; then $X^{\prime} \in \operatorname{Perf}_{\zeta}$ by Lemma 11, and $Y \in \operatorname{Perf}_{\zeta}$ as well. Let $\zeta^{\prime}=\zeta \cap J$. Then obviously $X^{\prime} \uparrow \zeta^{\prime}=Z^{\prime} \uparrow^{-1} \zeta^{\prime}$, so that $Y \uparrow \zeta^{\prime} \subseteq X^{\prime} \uparrow \zeta^{\prime}$ because $Y$ is stronger than $Z^{\prime}$. Hence the set $X=X^{\prime} \cap\left(Y \uparrow \zeta^{\prime} \uparrow^{-1} \zeta\right)$ belongs to Perf $f_{\zeta}$ by Lemmas 6 and 10, and $X\left\lceil\zeta^{\prime}=Y\left\lceil\zeta^{\prime}\right.\right.$. Finally, $X$ is stronger than $Z$, so $X$ forces $\Phi$.)

$\left({ }^{8}\right)$ The "additional" part of the theorem is not merely an example of the usual "restriction" argument because it is not excluded that $\xi \nsubseteq J$. However, the parameters $\mathbf{x}\lceil\xi$ enter the formula in quite a specific way: only the $\mathfrak{M}$-degree of $\mathbf{x}\lceil\xi$ rather than $\mathbf{x}\lceil\xi$ itself participates in the sentence. This makes it possible to use the homeomorphisms included in the definition of forcing conditions in Section 1.

The basic part of the theorem could be proved by a more general reasoning using Lemmas 6, 10, 11 of Section 1. 
We argue in $\mathfrak{M}$.

Both $X$ and $Y$ are members of Perf $f_{\zeta}$ satisfying $X\left\lceil\zeta^{\prime}=Y\left\lceil\zeta^{\prime}\right.\right.$, so there is, by Lemma 9 , a projection-keeping homeomorphism $H: X \stackrel{\text { onto }}{\longrightarrow} Y$ such that $x\left\lceil\zeta^{\prime}=H(x)\left\lceil\zeta^{\prime}\right.\right.$ for all $x \in X$. For each $\eta \in \mathbf{I S}_{\zeta}$, an associated projectionkeeping homeomorphism $H_{\eta}: X\left\lceil\eta \stackrel{\text { onto }}{\longrightarrow} Y\left\lceil\eta\right.\right.$ is defined by $H_{\eta}(x\lceil\eta)=H(x)\lceil\eta$ for all $x \in X$.

Furthermore, if $\zeta \subseteq \vartheta \in \boldsymbol{\Xi}$, then Lemma 11 defines the sets $X \uparrow^{-1} \vartheta$ and $Y \uparrow^{-1} \vartheta$ in $\operatorname{Perf}_{\vartheta}$ and a projection-keeping homeomorphism $H^{\vartheta}$ of $X \uparrow^{-1} \vartheta$ onto $Y \uparrow^{-1} \vartheta$ by $H^{\vartheta}\left(x^{\prime}\right)\left\lceil\zeta=H\left(x^{\prime}\lceil\zeta)\right.\right.$ and $H^{\vartheta}\left(x^{\prime}\right) \uparrow(\vartheta \backslash \zeta)=x^{\prime} \uparrow(\vartheta \backslash \zeta)$ for all $x^{\prime} \in X \uparrow^{-1} \vartheta$.

This allows us to define a total order isomorphism $\mathcal{H}=\mathcal{H}_{X Y}$ of $\mathbb{P}(X)$, the part of $\mathbb{P}$ stronger than $X$, onto $\mathbb{P}(Y)$, the part of $\mathbb{P}$ stronger than $Y$, as follows. Let $W \in \mathbb{P}(X)$. Then $\vartheta=\|W\| \supseteq \zeta$ and $W \subseteq X \uparrow^{-1} \vartheta$. We put $\mathcal{H}(W)=H^{\vartheta "} W$; then $\mathcal{H}(W) \in \operatorname{Perf}_{\vartheta}$ by Lemma 7 , hence $\mathcal{H}(W) \in \mathbb{P}(Y)$. This automorphism does not change projections outside $\zeta \backslash \zeta^{\prime}$, and therefore does not change the projection on $\vartheta \cap J$ because $\zeta^{\prime}=\zeta \cap J$.

We argue in the universe.

To avoid a mess with notation, assume that $X$ belongs to $G$, the $\mathbb{P}$-generic set already fixed. Applying the automorphism, we can obtain another set $G^{\prime}, \mathbb{P}$-generic over $\mathfrak{M}$, containing $Y$, such that the subsets $G_{X}=G \cap \mathbb{P}(X)$ and $G_{Y}^{\prime}=G^{\prime} \cap \mathbb{P}(Y)$ are connected by $\mathcal{H}$ as follows: $G_{Y}^{\prime}=\mathcal{H}^{\prime \prime} G_{X}$. Let $\mathbf{x}^{\prime} \in \mathcal{D}^{\mathbf{I}}$ be obtained from $G^{\prime}$ as $\mathbf{x}$ is from $G$ above.

Then $\mathfrak{M}[G]=\mathfrak{M}\left[G^{\prime}\right]$ since $\mathcal{H} \in \mathfrak{M}$. Furthermore, $\mathbf{x}\left\lceil J=\mathbf{x}^{\prime} \mid J\right.$ (by the "does not change projections" property above).

We also assert that $\mathfrak{M}\left[\mathbf{x}\lceil\xi]=\mathfrak{M}\left[\mathbf{x}^{\prime} \mid \xi\right]\right.$ for all $\xi \in \boldsymbol{\Xi}$. Suppose that this assertion has been proved. Then $\Phi$ interpreted by $G$ coincides with $\Phi$ interpreted by $G^{\prime}$. Therefore, by the choice of $X$ and $Y$, the sentence $\Phi$ is true in the model $\mathfrak{N}=\mathfrak{M}[G]$ and false in $\mathfrak{N}=\mathfrak{M}\left[G^{\prime}\right]$, a contradiction.

It remains to prove that $\mathfrak{M}\left[\mathbf{x}\lceil\xi]=\mathfrak{M}\left[\mathbf{x}^{\prime} \mid \xi\right]\right.$ for all $\xi \in \mathbf{\Xi}$. Let $\xi \in \mathbf{\Xi}$. The problem is that $\xi \cap \zeta$ may not be an initial segment of $\zeta$. To fix this, we put $\eta=\{j \in \zeta: \exists i \in \xi(j \leq i)\}$ and $\xi^{\prime}=\xi \cup \eta$. Lemma 22 implies $\mathfrak{M}\left[\mathbf{x}\lceil\xi]=\mathfrak{M}\left[\mathbf{x}\left\lceil\xi^{\prime}\right]\right.\right.$ and $\mathfrak{M}\left[\mathbf{x}^{\prime}\lceil\xi]=\mathfrak{M}\left[\mathbf{x}^{\prime}\left\lceil\xi^{\prime}\right]\right.\right.$. On the other hand, $\xi^{\prime} \cap \zeta=$ $\eta \in \mathbf{I S}_{\zeta}$, so that $\mathbf{x}^{\prime}\left\lceil\eta=H_{\eta}^{\#}(\mathbf{x} \uparrow \eta)\right.$ while $\mathbf{x}^{\prime} \uparrow\left(\xi^{\prime} \backslash \eta\right)=\mathbf{x} \uparrow\left(\xi^{\prime} \backslash \eta\right)$ by definition. We conclude that $\mathfrak{M}\left[\mathbf{x} \mid \xi^{\prime}\right]=\mathfrak{M}\left[\mathbf{x}^{\prime} \uparrow \xi^{\prime}\right]$, as required.

Preservation of cardinals. Let us prove that the cardinals $\aleph_{1}^{\mathfrak{M}}$ and sometimes $\aleph_{2}^{\mathfrak{M}}$ are preserved in the model $\mathfrak{N}=\mathfrak{M}[G]=\mathfrak{M}[\mathbf{x}]$. (The behaviour of other cardinals depends on the cardinal structure in $\mathfrak{M}$ and some cardinal characteristics of $\mathbf{I}$ in $\mathfrak{M}$. It is not our intention here to investigate this matter.) 
THEOREM 24. (1) $\aleph_{1}^{\mathfrak{M}}$ remains a cardinal in $\mathfrak{N}$.

(2) If $2^{\aleph_{0}}=\aleph_{1}$ in $\mathfrak{M}$ and every proper initial segment $J \in \mathfrak{M}, J \subseteq \mathbf{I}$, has cardinality card $J \leq \aleph_{1}^{\mathfrak{M}}$ in $\mathfrak{M}$ then $\aleph_{2}^{\mathfrak{M}}$ remains a cardinal in $\mathfrak{N}$.

Proof. (1) Let $f$ be a name of a function mapping $\omega$ to $\omega_{1}^{\mathfrak{M}}$. It would be enough, given $X_{0}^{-} \in \mathbb{P}$, to find a condition $X \in \mathbb{P}$ stronger than $X_{0}$ and a set $W \subseteq \omega_{1}^{\mathfrak{M}}$ countable in $\mathfrak{M}$ such that $X$ forces that the range of $f$ is included in $W$.

So let $X_{0} \in \mathbb{P}$.

We argue in $\mathfrak{M}$. Let $\xi_{0}=\left\|X_{0}\right\|$. We define the following objects:

1) a sequence $\zeta_{0} \subseteq \zeta_{1} \subseteq \zeta_{2} \subseteq \ldots$ of sets $\zeta_{m} \in \boldsymbol{\Xi}$ such that $\xi_{0} \subseteq \zeta_{0}$;

2) the set $\zeta=\bigcup_{m \in \omega} \zeta_{m} \in \boldsymbol{\Xi}$ and a $\zeta$-complete function $\phi: \omega \rightarrow \zeta$ such that $\phi(m) \in \zeta_{m}$ for all $m$;

3) for any $m$, a $\phi$-splitting system $\left\langle X_{u}: u \in 2^{m}\right\rangle$ of sets $X_{u} \in \operatorname{Perf}_{\zeta_{m}}$ such that $X_{\Lambda} \subseteq X_{0} \uparrow^{-1} \zeta_{0}$ and

(a) $\left.X_{u^{\wedge} e} \subseteq X_{u}\right|^{-1} \zeta_{m+1}$ for all $u \in 2^{m}$ and $e=0,1$;

(b) every set $X_{u}\left(u \in 2^{m}\right)$ has diameter diam $X_{u} \leq m^{-1}$;

(c) every condition $X_{u}\left(u \in 2^{m}\right)$ forces $f(m)=\varrho_{u}$ for a certain ordinal $\varrho_{u}$.

This solves the problem. Indeed, the sets $Y_{u}=X_{u} \uparrow^{-1} \zeta$ form a $\phi$-fusion sequence $\left({ }^{9}\right)$ in $\operatorname{Perf}_{\zeta}$, hence $X=\bigcap_{m \in \omega} \bigcup_{u \in 2^{m}} Y_{u} \in \operatorname{Perf}_{\zeta}$ by Theorem 15, and $X$ is stronger than $X_{0}$ by the construction. Finally, $X$ forces that the range of $f$ is a subset of a set $W=\left\{\varrho_{u}: u \in 2^{<\omega}\right\}$ countable in $\mathfrak{M}$.

So let us concentrate on the construction. Pick up a condition $X_{\Lambda}$ stronger than $X_{0}$ which decides the value $f(0)$, and put $\zeta_{0}=\left\|X_{\Lambda}\right\|$.

Suppose that $\phi\left\lceil m, \zeta_{m}\right.$, and the sets $X_{u}^{-}\left(u \in 2^{m}\right)$ have been defined. Let $u_{0} \in 2^{m}$. There exists a condition $Z \in \operatorname{Perf}_{\zeta^{\prime}}$ for some $\zeta^{\prime} \in \boldsymbol{\Xi}, \zeta^{\prime} \supseteq \zeta_{m}$, which is stronger than $X_{u_{0}}$, decides the value $f(m+1)$, and has diam $Z \leq$ $(m+1)^{-1}$. (We use Lemma 8 to fulfill the last inequality.) Let $Y_{u}^{\prime}=X_{u} \uparrow^{-1} \zeta^{\prime}$ for all $u \in 2^{m}$; then $\left\langle Y_{u}^{\prime}: u \in 2^{m}\right\rangle$ is a $\left(\phi\lceil m)\right.$-splitting system in Perf $\zeta_{\zeta^{\prime}}$ and $Z \subseteq Y_{u_{0}}^{\prime}$. Using Lemma 12 , we obtain a $\left(\phi\lceil m)\right.$-splitting system $\left\langle X_{u}^{\prime}: u \in\right.$ $\left.2^{m}\right\rangle$ in Perf $\zeta_{\zeta^{\prime}}$ such that $X_{u}^{\prime} \subseteq Y_{u}^{\prime}=X_{u} \Gamma^{-1} \zeta^{\prime}$ for all $u \in 2^{m}$ and the condition $X_{u_{0}}^{\prime}=Z$ decides the value $f(m+1)$.

Running this procedure $2^{m}$ times, we finally get a set $\zeta_{m+1} \in \boldsymbol{\Xi}$ satisfying $\zeta_{m+1} \supseteq \zeta_{m}$, and a $\left(\phi\lceil m)\right.$-splitting system $\left\langle X_{u}^{\prime}: u \in 2^{m}\right\rangle$ in $\operatorname{Perf}_{\zeta_{m+1}}$ such that $X_{u}^{\prime} \subseteq X_{u} \uparrow^{-1} \zeta_{m+1}$, diam $X_{u}^{\prime} \leq(m+1)^{-1}$, and $X_{u}^{\prime}$ decides the value $\underline{f}(m+1)$ for all $u \in 2^{m}$.

$\left({ }^{9}\right)$ We assume that $\operatorname{diam}\left(Z \uparrow^{-1} \zeta\right) \leq \operatorname{diam} Z$ whenever $Z \subseteq \mathcal{D}^{\xi}$ and $\xi \subseteq \zeta$. This suffices to prove S-3 for the sets $X_{u}$ by $\operatorname{diam} Y_{u} \leq \operatorname{diam} X_{u} \leq m^{-1}$ for $u \in 2^{m}$. 
At this moment, we define $\phi(m) \in \zeta_{m}$ appropriately, to ensure the final $\zeta$-completeness of $\phi$, and use Lemma 14 to obtain a $(\phi \uparrow(m+1))$-splitting system $\left\langle X_{u^{\prime}}: u^{\prime} \in 2^{m+1}\right\rangle$ in $\operatorname{Perf}_{\zeta_{m+1}}$ such that $X_{u^{\wedge} e} \subseteq X_{u}^{\prime} \subseteq X_{u} \uparrow^{-1} \zeta_{m+1}$ for all $u \in 2^{m}$ and $e=0,1$. This ends the recursive step of the construction.

(2) To prove $\aleph_{2}^{\mathfrak{N}}=\aleph_{2}^{\mathfrak{M}}$, it suffices to show that $\mathbb{P}$ does not have antichains of cardinality $>\aleph_{1}$ in $\mathfrak{M}$.

We argue in $\mathfrak{M}$. In particular, we have $\mathfrak{c}=\aleph_{1}$.

Let $\mathcal{A} \subseteq \mathbb{P}$ be a maximal antichain. The set $\mathbb{P}_{J}=\bigcup_{\zeta \in \Xi, \zeta \subseteq J}$ Perf ${ }_{\zeta}$ has cardinality $\operatorname{card} \mathbb{P}_{J} \leq \aleph_{1}$ (in fact $=$, of course) for any proper (i.e. other than $\mathbf{I}$ itself) initial segment $J \subseteq \mathbf{I}$ by the assumptions of the theorem. Therefore there exists an initial segment $J \subseteq \mathbf{I}$ of cardinality card $J \leq \aleph_{1}$ such that $\mathcal{A}^{\prime}=\mathcal{A} \cap \mathbb{P}_{J}$ is a maximal antichain in $\mathbb{P}_{J}$.

It remains to check that $\mathcal{A}=\mathcal{A}^{\prime}$. Suppose on the contrary that $X \in$ $\mathcal{A} \backslash \mathcal{A}^{\prime}$. Let $\zeta=\|X\|$ and $\eta=\zeta \cap J$. Then $X \in \operatorname{Perf}_{\zeta}$ and $Y=X\left\lceil\eta \in \operatorname{Perf}_{\eta}\right.$ and $\in \mathbb{P}_{J}$ by Lemma 6 . Therefore there exist sets $Z^{\prime} \in \mathcal{A}^{\prime}$ and $Z \in \mathbb{P}_{J}$ such that $Z$ is stronger than both $Z^{\prime}$ and $Y$. We come to a contradiction if we prove that $Z$ and $X$ are compatible in $\mathbb{P}$.

Let $\xi=\|Z\|$, so that $\eta \subseteq \xi \subseteq J$, and $\vartheta=\xi \cup \zeta$. Then $X^{\prime}=X \uparrow^{-1} \vartheta \in$ $\operatorname{Perf}_{\vartheta}$ by Lemma 11. The set $\xi=\vartheta \cap J$ is an initial segment in $\vartheta$ and obviously $X^{\prime} \mid \xi=Y \uparrow^{-1} \xi$; hence $Z \subseteq X^{\prime} \mid \xi$. Now $X^{\prime \prime}=X^{\prime} \cap\left(Z \uparrow^{-1} \vartheta\right) \in \operatorname{Perf}_{\vartheta}$ by Lemma 10 . But $X^{\prime \prime}$ is stronger than both $Z$ and $X$.

Continuous functions. We put $\mathbb{F}_{\zeta}=\left(\operatorname{Cont}_{\zeta}\right)^{\mathfrak{M}}$ for $\zeta \in \boldsymbol{\Xi}$. It is a principal property of several forcing notions (including Sacks forcing and for instance random forcing) that reals in the generic extensions can be obtained by application of continuous functions (having a code) in the ground model, to generic sequences of reals. As we shall prove, this is also a property of the generic models considered here.

Every $F \in \mathbb{F}_{\zeta}$ is a countable subset of $\mathcal{D}^{\zeta} \times \omega^{\omega}$ in the universe, but since the domain of $F$ in $\mathfrak{M}$ is the compact set $\mathcal{D}^{\zeta}$, the topological closure $F^{\#}$ is a continuous function mapping $\mathcal{D}^{\zeta}$ into the reals in the universe.

By "reals" we understand elements of the set $\mathcal{N}=\omega^{\omega}$, as usual.

TheOREM 25. Let $J \in \mathfrak{M}$ be an initial segment of $\mathbf{I}$ and $r$ a real in $\mathfrak{M}\left[\mathbf{x}\lceil J]\right.$. There exists a set $\zeta \in \mathbf{\Xi}, \zeta \subseteq J$, and a function $F \in \mathbb{F}_{\zeta}$ such that $r=F^{\#}(\mathbf{x}\lceil\zeta)$.

(The equality is obviously absolute for any model containing $r, \mathbf{x} \mid \zeta, F$.)

Proof. Let $\underline{r}$ be a name for the real $r$, containing an explicit absolute construction of $r$ from $\mathbf{x}\left\lceil J\right.$ and some parameter $p \in \mathfrak{M}$. Let $X_{0} \in \mathbb{P}$.

We argue in $\mathfrak{M}$. Let $\xi_{0}=\left\|X_{0}\right\|$.

By Theorem 23 the forcing of statements about $\underline{r}$ can be reduced to $J$ : if $X \in \operatorname{Perf}_{\zeta}$ forces $\underline{r}(m)=k$ then $X\lceil(\zeta \cap J)$ also forces $\underline{r}(m)=k$. 
Having this in mind and arguing as in the proof of Theorem 24, one gets a system of objects satisfying 1), 2), 3), with the following corrections: in 1), additionally, $\zeta_{m} \subseteq J$-therefore $\zeta \subseteq J$, and in 3)(c), every condition $X_{u}$, $u \in 2^{m}$, forces $\underline{r}(m)=k_{u}$ for some $k_{u} \in \omega$. We set $Y_{u}=X_{u} \uparrow^{-1} \zeta$ for all $u \in 2^{<\omega}$.

Define a continuous function $F^{\prime}$ on the set $X=\bigcap_{m} \bigcup_{u \in 2^{m}} Y_{u} \in \operatorname{Perf}_{\zeta}$ as follows. Let $x \in X$ and $m \in \omega$. There exists a unique $u \in 2^{m}$ such that $x \in Y_{u}$. We put $F^{\prime}(x)(m)=k_{u}$. The function $F^{\prime}$ can be extended to a function $F \in \operatorname{Cont}_{\zeta}$ (that is, defined on the whole $\mathcal{D}^{\zeta}$ ). Then $X$ forces $\underline{r}=F^{\prime \#}(\mathbf{x} \mid \zeta)=F^{\#}(\mathbf{x}\lceil\zeta)$.

5. Reals in the iterated Sacks extensions. Theorem 25 practically reduces properties of reals in $\mathbb{P}$-generic extensions to properties of continuous functions in the ground model. To demonstrate how Theorem 25 works we prove several lemmas on reals in a $\mathbb{P}$-generic model $\mathfrak{N}=\mathfrak{M}[G]$. Section 3 will be taken as a source of different properties of continuous functions in the ground model.

As an application, Theorem 3 will be proved.

At the end of this section, it will be demonstrated that the models we consider are iterated Sacks extensions.

We keep the notation of the previous section.

In fact, this section should start with Lemma 22, which, indeed, was necessary for the proof of Theorem 23.

Lemma 26. Suppose that $J \in \mathfrak{M}$ is an initial segment in $\mathbf{I}$ and $i \in \mathbf{I} \backslash J$. Then $\mathbf{a}_{i} \notin \mathfrak{M}[\mathbf{x}\lceil J]$.

Pro of. Otherwise, by Theorem 25 , there exist $\zeta \in \boldsymbol{\Xi}$, a function $F \in \mathbb{F}_{\xi}$, where $\xi=\zeta \cap J$, and a condition $X \in \mathbb{P}_{\zeta}$ which forces $\mathbf{a}_{i}=F^{\#}(\mathbf{x}\lceil\xi)$.

We argue in $\mathfrak{M}$. We have $x(i)=F(x \mid \xi)$ for all $x \in X$. (Indeed, otherwise there exist $m \in \omega$ and a condition $Y \subseteq X, Y \in \operatorname{Perf}_{\zeta}$, such that $x(i)(m)=0$ but $F(x\lceil\xi)(m)=1$, or vice versa, for all $x \in Y$, by Lemma 8. One easily gets a contradiction with the choice of the condition $X$.) Thus the coordinate function $C_{i}$ reduces to $\xi$ on $X$, contradicting Theorem 20(2) because $i \notin \xi$.

Corollary 27. If $i \neq j$ then $\mathbf{a}_{i} \neq \mathbf{a}_{j}$ and even $\mathfrak{M}\left[\mathbf{a}_{i}\right] \neq \mathfrak{M}\left[\mathbf{a}_{j}\right]$.

Lemma 28. If $\xi \in \boldsymbol{\Xi}$ and $r$ is a real in $\mathfrak{N}$ such that $\mathbf{a}_{i} \in \mathfrak{M}[r]$ for all $i \in \xi$ then the indexed set $\mathbf{x}\left\lceil\xi=\left\langle\mathbf{a}_{i}: i \in \xi\right\rangle\right.$ belongs to $\mathfrak{M}[r]$.

Proof. Otherwise, by Theorem 25 , there exist a set $\zeta \in \boldsymbol{\Xi}$ such that $\xi \subseteq \zeta$, a function $F \in \mathbb{F}_{\zeta}$, and a condition $X \in \mathbb{P}_{\zeta}$ which forces that $\mathbf{a}_{i} \in \mathfrak{M}\left[F^{\#}(\mathbf{x} \backslash \zeta)\right]$ for each $i \in \xi$, but also forces $\mathbf{x}\left\lceil\xi \notin \mathfrak{M}\left[F^{\#}(\mathbf{x} \backslash \zeta)\right]\right.$. One can assume, by Lemma 22 , that $\xi$ is an initial segment of $\zeta$. 
We argue in $\mathfrak{M}$. It follows from Theorem $20(3,4)$ that there exists a condition $Y \in \operatorname{Perf}_{\zeta}, Y \subseteq X$, such that either $F$ captures each $i \in \xi$ on $Y$, or, for some $i \in \xi, F$ reduces to $\eta=\zeta \cap[\nsupseteq i]$ on $Y$.

In the "either" case, there exists a continuous function $H$ : reals $\rightarrow$ $\mathcal{D}^{\xi}$ such that $x \mid \xi=H(F(x))$ for all $x \in Y$. We conclude that $Y$ forces $\mathbf{x}\left\lceil\xi=H^{\#}\left(F^{\#}(\mathbf{x} \backslash \zeta)\right)\right.$, so forces $\mathbf{x} \mid \xi \in \mathfrak{M}\left[F^{\#}(\mathbf{x} \mid \zeta)\right]$, contradicting the choice of $X$.

In the "or" case, $Y$ forces $F^{\#}(\mathbf{x} \backslash \zeta) \in \mathfrak{M}\left[\mathbf{x}\lceil\eta]\right.$, in particular, forces $\mathbf{a}_{i} \in$ $\mathfrak{M}[\mathbf{x}\lceil\eta]$. We observe that $J=[\nsupseteq i]=\{j \in \mathbf{I}: j \nsupseteq i\}$ is an initial segment in $\mathbf{I}$ and $\eta \subseteq J \in \mathfrak{M}$, so $Y$ forces $\mathbf{a}_{i} \in \mathfrak{M}[\mathbf{x}\lceil J]$, contradicting Lemma 26 because $i \notin J$.

Lemma 29. If $J \in \mathfrak{M}$ is an initial segment of $\mathbf{I}$, and $r$ is a real in $\mathfrak{N}$, then either $r \in \mathfrak{M}\left[\mathbf{x}\lceil J]\right.$ or there is $i \notin J$ such that $\mathbf{a}_{i} \in \mathfrak{M}[r]$.

Proof. Otherwise, by Theorem 25, there exist $\zeta \in \boldsymbol{\Xi}$, a function $F \in \mathbb{F}_{\zeta}$, and a condition $X \in \mathbb{P}_{\zeta}$ which forces $F^{\#}(\mathbf{x}\lceil\zeta) \notin \mathfrak{M}[\mathbf{x}\lceil J]$ and forces $\mathbf{a}_{i} \notin \mathfrak{M}\left[F^{\#}(\mathbf{x} \backslash \zeta)\right]$ for all $i \in \mathbf{I} \backslash J$.

We argue in $\mathfrak{M}$. Set $\xi=\zeta \cap J$. Then $\xi$ is an initial segment of $\zeta$. It follows from Theorem 20(5) that there is $Y \in \operatorname{Perf}_{\zeta}, Y \subseteq X$, such that either $F$ reduces to $\xi$ on $Y$ or $F$ captures some $i \in \zeta \backslash \xi$ on $Y$.

In the "either" case, $Y$ forces $F^{\#}(\mathbf{x}\lceil\zeta) \in \mathfrak{M}[\mathbf{x}\lceil\xi]$ as above, a contradiction since $\xi \subseteq J$. In the "or" case, $Y$ forces $\mathbf{a}_{i}=\mathbf{x}(i) \in \mathfrak{M}\left[F^{\#}(\mathbf{x}\lceil\zeta)]\right.$ as above, a contradiction because $i \notin J$.

The "discrete" case and the degrees of constructibility. In this subsection we consider a special but quite important class of sets I which admit a complete description of the degrees of $\mathfrak{M}$-constructibility of reals in the extension in terms of initial segments of $\mathbf{I}$.

We keep the notation introduced above.

Definition. A (partially ordered) set $\mathbf{I} \in \mathfrak{M}$ is $\mathfrak{M}$-discrete iff all initial segments of $\mathbf{I}$ belong to $\mathfrak{M}$.

For instance $\mathbb{Z}$ (the integers), ordinals, and inverse ordinals are discrete. $\mathbb{Q}$ (the rationals) is not an $\mathfrak{M}$-discrete set. An infinite set with the empty order is not discrete.

For a real $r \in \mathfrak{N}$, we set $\mathbf{I}_{r}=\left\{i \in \mathbf{I}: \mathbf{a}_{i} \in \mathfrak{M}[r]\right\}$; then $\mathbf{I}_{r} \in \mathfrak{M}[G]$ is an initial segment of $\mathbf{I}$ by Lemma 22. The following theorem shows, in particular, that in the case of a discrete set $\mathbf{I}$ the $\mathfrak{M}$-degrees of reals in $\mathfrak{M}$ admit a description in terms of initial segments of $\mathbf{I}$ having countable cofinality in $\mathfrak{M}$. 
Theorem 30. Suppose that $\mathbf{I}$ is $\mathfrak{M}$-discrete. Then

(1) For each real $r \in \mathfrak{N}, \mathbf{I}_{r}$ belongs to $\mathfrak{M}$ and has countable cofinality in $\mathfrak{M}$.

(2) If $\zeta \in \mathbf{\Xi}$ is cofinal in $\mathbf{I}_{r}$ then $\mathfrak{M}[r]=\mathfrak{M}\left[\mathbf{x}\lceil\zeta]\right.$. In particular, if $i_{0}$ is the largest element of $\mathbf{I}_{r}$ then $\mathfrak{M}[r]=\mathfrak{M}\left[\mathbf{a}_{i_{0}}\right]$.

(3) For all reals $r, r^{\prime} \in \mathfrak{N}, r \in \mathfrak{M}\left[r^{\prime}\right]$ iff $\mathbf{I}_{r} \subseteq \mathbf{I}_{r^{\prime}}$.

(We understand countable cofinality so that a p.o. set $J$ is countably cofinal iff there exists a countable or finite subset $\xi \subseteq J$ such that each $j \in J$ is $\leq$ than some $i \in \xi$. In particular, sets having the largest element are of countable cofinality.)

Proof. (1) First of all, $\mathbf{I}_{r} \in \mathfrak{M}$ since $\mathbf{I}$ is $\mathfrak{M}$-discrete. We have $r \in \mathfrak{M}\left[\mathbf{x}\left\lceil\mathbf{I}_{r}\right]\right.$ by Lemma 29. Hence $r \in \mathfrak{M}\left[\mathbf{x}\lceil\xi]\right.$ for some $\xi \in \mathbf{\Xi}, \xi \subseteq \mathbf{I}_{r}$ by Theorem 25. It follows that $\mathbf{a}_{i} \in \mathfrak{M}\left[\mathbf{x}\lceil\xi]\right.$ whenever $i \in \mathbf{I}_{r}$. Therefore $\xi$ is cofinal in $\mathbf{I}_{r}$ by Lemma $26\left({ }^{10}\right)$.

(2) Let $\zeta \in \boldsymbol{\Xi}$ be cofinal in $\mathbf{I}_{r}$. Then $\mathbf{x}\lceil\zeta \in \mathfrak{M}[r]$ by Lemma 28. To prove the converse, we recall that $r \in \mathfrak{M}\left[\mathbf{x}\lceil\xi]\right.$ for some $\xi \in \mathbf{\Xi}, \xi \subseteq \mathbf{I}_{r}$, see above. Then $r \in \mathfrak{M}\left[\mathbf{x}\lceil\zeta]\right.$ by Lemma 22 since $\zeta$ is cofinal in $\mathbf{I}_{r}$.

(3) Suppose that $\mathbf{I}_{r} \subseteq \mathbf{I}_{r^{\prime}}$. As above there exists $\xi \in \boldsymbol{\Xi}, \xi \subseteq \mathbf{I}_{r}$, such that $r \in \mathfrak{M}\left[\mathbf{x}\lceil\xi]\right.$. Then we have $\xi \subseteq \mathbf{I}_{r^{\prime}}$ as well, hence $\mathbf{x}\left\lceil\xi \in \mathfrak{M}\left[r^{\prime}\right]\right.$ by Lemma 28.

A model in which every new real collapses $\kappa$ to $\aleph_{1}$. To prove Theorem 3 , we suppose that $\kappa$ is an uncountable cardinal in $\mathfrak{M}$, a model of $\mathrm{V}=\mathrm{L}$. Let $\mathbf{I}=\kappa^{*}$ (i.e. $\kappa$ with the inverse order). Obviously $\mathbf{I}$ is $\mathfrak{M}$-discrete.

Note that $\aleph_{1}^{\mathfrak{M}}$ remains a cardinal in the $\mathbb{P}_{\mathbf{I}^{-}}$generic extension $\mathfrak{N}=\mathfrak{M}[G]$ by Theorem 24. Let us prove that $\kappa$ admits a bijection on $\aleph_{1}^{\mathfrak{M}}$ in any submodel $\mathfrak{M}[r]$, where $r$ is a real in $\mathfrak{N}=\mathfrak{M}[G], r \notin \mathfrak{M}$.

The initial segment $\mathbf{I}_{r}$ is nonempty by Theorem 30(3) as $r \notin \mathfrak{M}$. We observe that $\mathbf{I}_{r} \in \mathfrak{M}$ since all initial segments of $\mathbf{I}=\kappa^{*}$ belong to $\mathfrak{M}$. Furthermore, card $\mathbf{I}_{r}=\kappa$ in $\mathfrak{M}$.

On the other hand, all reals $\mathbf{a}_{i}$ are pairwise different by Corollary 27, so we have at least $\kappa$ different reals in $\mathfrak{M}[r]$. But $\mathfrak{M}[r]=(\mathrm{L}[r])^{\mathfrak{N}}$.

The "Sacksness". We prove that the model $\mathfrak{N}$ is a sort of iterated Sacks generic extension of $\mathfrak{M}$. This separate result will not be used below.

Theorem 31. Every $\mathbf{a}_{i}$ is Sacks generic over the model $\mathfrak{M}\left[\left.\mathbf{x}\right|_{<i}\right]$.

Before the proof starts, we have to present one more construction of forcing conditions. Perhaps, Section 1 would be a more suitable place, but we decided to introduce it here because it is used only to prove Theorem 31 .

$\left({ }^{10}\right)$ Conversely, if $J \in \mathfrak{M}$ is a countably cofinal initial segment of $\mathbf{I}$ in $\mathfrak{M}$ then one easily proves that $J=\mathbf{I}_{r}$ for a real $r \in \mathfrak{N}$. 
We consider trees $T \subseteq 2^{<\omega}$. Let a perfect tree be any (nonempty) tree $T \subseteq 2^{<\omega}$ such that the set $B(T)=\left\{t \in T: t^{\wedge} 0 \in T \& t^{\wedge} 1 \in T\right\}$ of all splitting points of $T$ is cofinal in $T$. Suppose $T$ is such a tree. We define the following objects.

- $[T]=\left\{a \in 2^{\omega}: \forall m(a\lceil m \in T)\}\right.$, a perfect set is $\mathcal{D}=2^{\omega}$.

- An order isomorphism $\beta_{T}: 2^{<\omega} \stackrel{\text { onto }}{\longrightarrow} B(T)$. We define $\beta_{T}(u) \in B(T)$ for every $u \in 2^{<\omega}$ by induction on $\operatorname{dom} u$, putting $\beta_{T}\left(u^{\wedge} e\right)$ to be the least element $s \in B(T)$ such that $\beta_{T}(u)^{\wedge} e \subseteq s$, for $e=0,1$.

- A homeomorphism $H_{T}: \mathcal{D} \stackrel{\text { onto }}{\longrightarrow}[T]$ by $H_{T}(a)=\bigcup_{m \in \omega} \beta_{T}(a\lceil m)$ for all $a \in \mathcal{D}$.

LEMma 32. Assume that $i$ is the largest element in $\zeta \in \boldsymbol{\Xi}, \eta=\zeta \backslash\{i\}$, $Y \in \operatorname{Perf}_{\eta}, y \mapsto T(y)$ continuously maps $Y$ into $\mathcal{P}\left(2^{<\omega}\right)$, and $T(y)$ is a perfect tree for all $y \in Y$. Then the set $X=\left\{x \in \mathcal{D}^{\zeta}: x\lceil\eta \in Y \& x(i) \in\right.$ $[T(x\lceil\eta)]\}$ belongs to $\operatorname{Perf}_{\zeta}$.

Proof of the lemma. The set $Z=Y \uparrow^{-1} \zeta$ belongs to Perf $f_{\zeta}$ by Lemma 11, so, by Lemma 7, it suffices to define a projection-keeping homeomorphism $H: Z \stackrel{\text { onto }}{\longrightarrow} X$. Let $z \in Z$. Then $y=z\lceil\eta \in Y$ while $a=z(i) \in \mathcal{D}$ is arbitrary. We define $x=H(z) \in \mathcal{D}^{\zeta}$ so that $x\left\lceil\eta=y\right.$ and $x(i)=H_{T(y)}(a)$. Then $H$ maps $Z$ onto $X$ because every $H_{T(y)}$ maps $\mathcal{D}$ onto $[T(y)]=\{x(i)$ : $x \in X \& x\lceil\eta=y\} . H$ is $1-1$ since each $H_{T}$ is $1-1$, and $H$ is continuous since so is the map $y \mapsto T(y)$. It remains to prove that $H$ is projection-keeping, i.e. $z_{0}\left|\xi=z_{1}\right| \xi \Leftrightarrow H\left(z_{0}\right)\left|\xi=H\left(z_{1}\right)\right| \xi$ for all $z_{0}, z_{1} \in Z$ and $\xi \in \mathbf{I S}_{\zeta}$. If $i \notin \xi$ then $\xi \subseteq \eta$ and $z\lceil\xi=H(z) \mid \xi$ by definition. If $i \in \xi$ then $\xi=\zeta$, so the result is obvious as well.

Proof of Theorem 31. Suppose that $S \in \mathfrak{M}\left[\left.\mathbf{x}\right|_{<i}\right]$ is, in $\mathfrak{M}\left[\left.\mathbf{x}\right|_{<i}\right]$, a dense subset in the collection of all perfect subsets of $\mathcal{D}$; we have to prove that $\mathbf{a}_{i} \in P^{\#}$ for some $P \in S$. Assume on the contrary that a condition $X_{0} \in G \cap \mathbb{P}_{\zeta}(\zeta \in \boldsymbol{\Xi})$ forces the opposite. Since the forced statement is relativized to $\mathfrak{M}\left[\left.\mathbf{x}\right|_{\leq i}\right]$, we may assume that $\zeta \subseteq[\leq i]$ by Theorem 23 . We can also suppose that $i \in \zeta$, so that $i$ is a maximal element in $\zeta$. Let $\eta=\zeta \cap[<i]=\zeta \backslash\{i\} ; \eta$ is an initial segment in $\zeta$.

We argue in $\mathfrak{M}$.

Note that the set $D(y)=D_{X_{0} y}(i)=\left\{x(i): x \in X_{0} \& x\lceil\eta=y\}\right.$ is a perfect subset of $\mathcal{D}=2^{\omega}$ for all $y \in Y_{0}=X_{0}\left\lceil\eta\right.$ by property P-1 of $X_{0}$ (see Proposition 4).

We argue in $\mathfrak{M}\left[\mathbf{x} \uparrow_{<i}\right]$.

We observe that $\mathbf{y}=\mathbf{x}\left\lceil\eta \in Y_{0}^{\#}\right.$. Therefore $D^{\#}(\mathbf{y})=D_{X_{0} \# \mathbf{y}}(i)$ is a perfect set. Thus there exists a set $P \in S$ such that $P \subseteq D^{\#}(\mathbf{y})$.

By the assumption, $\mathbf{a}_{i}=\mathbf{x}(i) \notin P^{\#}$. 
We put $T^{*}=\left\{p\lceil m: p \in P \& m \in \omega\}\right.$. Then $T^{*}$ is a perfect tree and $P=\left[T^{*}\right]$. By Theorem 25, there exist, in $\mathfrak{M}, \xi \in \boldsymbol{\Xi}$ such that $\xi \subseteq[<i]$, and a continuous map $y \mapsto T(y)$ of $\mathcal{D}^{\xi}$ into $\mathcal{P}\left(2^{<\omega}\right)$ such that $T^{*}=T^{\#}(\mathbf{x}\lceil\xi)$. We can assume that $\xi \subseteq \zeta$ (otherwise put $\zeta^{\prime}=\zeta \cup \xi$ and $X_{0}^{\prime}=X_{0} \uparrow^{-1} \zeta^{\prime}$ in $\mathfrak{M}$, etc.). Then $\xi \subseteq \eta$, so it can be assumed that simply $\xi=\eta$. Then $T^{*}=T^{\#}(\mathbf{y})$, so that $\left[T^{\#}(\mathbf{y})\right]=P \subseteq D^{\#}(\mathbf{y})$.

The statement " $T^{\#}(\mathbf{y})$ is a perfect tree, $\left[T^{\#}(\mathbf{y})\right] \in S$, and $\left[T^{\#}(\mathbf{y})\right] \subseteq$ $D^{\#}(\mathbf{y})$ " is relativized to $\mathfrak{M}[\mathbf{y}]=\mathfrak{M}[\mathbf{x}\lceil\eta]$; therefore it is forced by a condition $Y_{1} \in G$ stronger than $Y_{0}$ and such that $\xi=\left\|Y_{1}\right\| \subseteq[<i]$, by Theorem 23 . As above, we can assume that in fact $\xi=\eta$, so that $Y_{1} \subseteq Y_{0}$.

We argue in $\mathfrak{M}$.

The set $B=\left\{y \in Y_{1}: T(y)\right.$ is a perfect tree and $\left.[T(y)] \subseteq D(y)\right\}$ is a subset of $Y_{1}$ of a finite Borel level because $T$ is continuous. Therefore, by Corollary 17, we have either $Y \subseteq B$ or $Y \cap B=\emptyset$ for a set $Y \in \operatorname{Perf}_{\eta}$, $Y \subseteq Y_{1}$.

Suppose that $Y \cap B=\emptyset$. Then by Shoenfield $Y$ would force that either $T^{\#}(\mathbf{y})$ is not a perfect tree or $\left[T^{\#}(\mathbf{y})\right] \nsubseteq D^{\#}(\mathbf{y})$, contrary to the choice of $Y_{1}$. Therefore $Y \subseteq B$. In particular, $T(y)$ is a perfect tree for all $y \in Y$. It follows that the set $X=\left\{x \in \mathcal{D}^{\zeta}: x\lceil\eta \in Y \& x(i) \in[T(x\lceil\eta)]\}\right.$ belongs to Perf $_{\zeta}$ by Lemma 32. Furthermore, $[T(y)] \subseteq D(y)=D_{X_{0} y}(i)$ for all $y \in Y$, so that $X \subseteq X_{0}$. Since $X$ is also stronger than $Y_{1}, X$ forces everything which is forced by $X_{0}$ and/or $Y_{1}$, and everything which logically follows from the mentioned. In particular, since $X_{0}$ forces that $\mathbf{a}_{i}$ does not belong to a set in $S$ while $Y_{1}$ forces that $\left[T^{\#}(\mathbf{y})\right] \in S$, we conclude that $X$ forces $\mathbf{a}_{i} \notin\left[T^{\#}(\mathbf{y})\right]$. It follows that $X$ forces $\mathbf{a}_{i} \notin D_{X^{\#} \mathbf{y}}(i)$ because by definition $D_{X y}(i)=[T(y)]$. This means that $X$ forces $\mathbf{x} \uparrow \zeta \notin X^{\#}$ (indeed, $\mathbf{x} \uparrow \zeta=$ $\left.\mathbf{y} \cup\left\{\left\langle i, \mathbf{a}_{i}\right\rangle\right\}\right)$, a contradiction.

The next proposition (not to be proved here as it does not essentially contribute to our main results in the next section) gives a more substantial characterization of the models we consider.

Proposition 33. If $\mathbf{I}=\lambda$ is an ordinal in $\mathfrak{M}$ then $\mathbb{P}_{\mathbf{I}}$-generic extensions of $\mathfrak{M}$ are equal to countable support iterated Sacks extensions of $\mathfrak{M}$ of length $\lambda$. If $\mathbf{I}$ is an unordered set of cardinality $\kappa$ in $\mathfrak{M}$ then $\mathbb{P}_{\mathbf{I}^{-} \text {-generic extensions }}$ of $\mathfrak{M}$ are equal to generic extensions of $\mathfrak{M}$ via the countable support product of $\kappa$ copies of the Sacks forcing.

6. Non-Glimm-Effros equivalence relations. This section presents the proof of Theorems 1 and 2. The proofs differ in some detail, but also have much in common, in particular are based on several facts of general nature. Therefore we start with those general properties of the iterated Sacks models, and then detail the reasoning. 
We keep the notation $\left(\mathbb{P}, \boldsymbol{\Xi}, \mathbb{P}_{\zeta}\right.$ for $\zeta \in \mathbf{\Xi}, \mathfrak{M}, G, \mathbf{x}=\left\langle\mathbf{a}_{i}: i \in \mathbf{I}\right\rangle \in \mathcal{D}^{\mathbf{I}}$, $\mathfrak{N}=\mathfrak{M}[G]=\mathfrak{M}[\mathbf{x}]$ - the extension, etc.) of the preceding sections, but assume the following in addition:

(i) $\mathfrak{M}$, the ground model, satisfies the axiom of constructibility $\mathrm{V}=\mathrm{L}$.

(ii) $\mathbf{I}$ is an $\mathfrak{M}$-discrete set, i.e. all initial segments of $\mathbf{I}$ belong to $\mathfrak{M}$.

Fix a set $G \subseteq \mathbb{P}, \mathbb{P}$-generic over $\mathfrak{M}$, and consider the $\Sigma_{2}^{1}$ equivalence relation $\mathrm{C}$ defined on the reals by

$$
x \subset y \quad \text { iff } \quad \mathrm{L}[x]=\mathrm{L}[y],
$$

in the model $\mathfrak{N}=\mathfrak{M}[G]=\mathfrak{M}[\mathbf{x}]=\mathfrak{M}\left[\left\langle\mathbf{a}_{i}: i \in \mathbf{I}\right\rangle\right]$. Notice that C-equivalence classes, degrees of constructibility of reals, and degrees of $\mathfrak{M}$-constructibility of reals - are one and the same thing in $\mathfrak{N}$ by (i).

We start with a consequence of the "additional" part of Theorem 23.

A set $S$ of reals is called C-invariant if $x$ C $y$ implies $x \in S \Leftrightarrow y \in S$ for any two reals $x, y$. We say that a variable $v$ is C-invariant in a formula $\varphi(v)$ if it enters the formula only through the expression $\mathrm{L}[v]$.

Proposition 34. Suppose that $\xi \in \boldsymbol{\Xi}, J$ is an initial segment of $\mathbf{I}$, and the variable $v$ is $\mathbf{C}$-invariant in $\varphi(\mathbf{x}\lceil J, v, \alpha)$, a formula containing only $\mathbf{x}\lceil J$ and sets in $\mathfrak{M}$ as parameters. Then, for every ordinal $\lambda \in \mathfrak{M}$, the set $\Omega_{\lambda}=\{\alpha<\lambda: \mathfrak{N}=\varphi(\mathbf{x}\lceil J, \mathbf{x}\lceil\xi, \alpha)\}$ belongs to $\mathfrak{M}[\mathbf{x}\lceil J]$.

Proof. We have

$$
\Omega_{\lambda}=\{\alpha<\lambda: \exists X \in G(\|X\| \subseteq J \& X \text { forces } \varphi(\mathbf{x}\lceil J, \mathbf{x}\lceil\xi, \alpha))\}
$$

by Theorem 23 . Therefore, since $J \in \mathfrak{M}$ by (ii), it suffices to prove that

$$
\{X \in G:\|X\| \subseteq J\}=\left\{X \in \mathbb{P}:\|X\| \subseteq J \& \mathbf{x} \uparrow\|X\| \in X^{\#}\right\} .
$$

The nontrivial direction is $\supseteq$, so assume that $\vartheta \subseteq J, X \in \mathbb{P}_{\vartheta}$, and $\mathbf{x}\left\lceil\vartheta \in X^{\#}\right.$, and prove that $X \in G$. Suppose on the contrary that some $Z \in G$ forces the opposite, and also forces that $\mathbf{x} \uparrow \vartheta \in X^{\#}$.

One may assume that $\vartheta \subseteq \zeta=\|Z\|$. Then $X \in \operatorname{Perf}_{\vartheta}$ and $Z \in \operatorname{Perf}_{\zeta}$ in $\mathfrak{M}$. Lemma 8 yields a set $Z^{\prime} \subseteq Z, Z^{\prime} \in \operatorname{Perf}_{\zeta}$, such that either $Z^{\prime} \uparrow \vartheta \subseteq X$ or $\left(Z^{\prime} \uparrow \vartheta\right) \cap X=\emptyset$. In the first case $Z^{\prime}$ is stronger than $X$, so $Z^{\prime}$ forces that $X$ belongs to $G$, a contradiction. In the second case, $Z^{\prime}$ forces that $\mathbf{x} \mid \vartheta \notin X^{\#}$, a contradiction as well.

Applications of order automorphisms. An ordinal does not admit a nontrivial order automorphism. However, both nonlinear wellfounded order relations and nonwellordered linear orders do admit one. We exploit the effect of existence of different but indistinguishable C-classes $\left[\mathbf{a}_{i}\right]_{\mathrm{C}}=\left\{r: r \mathrm{C} \mathbf{a}_{i}\right\}$ of reals $\mathbf{a}_{i}$, available in the case when $\mathbf{I}$ has a nontrivial order automorphism. 
Proposition 35. Assume that $J$ is an initial segment of $\mathbf{I}, h \in \mathfrak{M}$ is an order automorphism of $\mathbf{I}, h\left\lceil J\right.$ is the identity, $i \in \mathbf{I}, h(i)=i^{\prime}$, and $A \in \mathfrak{N}$ is a set of reals definable in $\mathfrak{N}$ by a formula with only $\mathbf{x}\lceil J$ and sets in $\mathfrak{M}$ as parameters. Then, in $\mathfrak{N}, A \cap\left[\mathbf{a}_{i}\right]_{\mathcal{C}}=\emptyset$ iff $A \cap\left[\mathbf{a}_{i^{\prime}}\right]_{\mathrm{C}}=\emptyset$.

Proof. Let $A=\{r: \psi(\mathbf{x}\lceil J, r)\}$ in $\mathfrak{N}$, where $\psi$ contains only $\mathbf{x}\lceil J$ and sets in $\mathfrak{M}$ as parameters.

Let $\varphi\left(\mathbf{x}\lceil J, r)\right.$ be the formula $\exists r^{\prime}\left(r \subset r^{\prime} \& \psi\left(\mathbf{x}\left\lceil J, r^{\prime}\right)\right)\right.$.

Assume on the contrary that e.g. $A \cap\left[\mathbf{a}_{i}\right]_{\mathcal{C}} \neq \emptyset$ but $A \cap\left[\mathbf{a}_{i^{\prime}}\right]_{\mathcal{C}}=\emptyset$ in $\mathfrak{N}$. This means that, in $\mathfrak{N}, \varphi\left(\mathbf{x}\lceil J, r)\right.$ is true for any $r \in\left[\mathbf{a}_{i}\right]_{\mathcal{C}}$ and false for any $r \in\left[\mathbf{a}_{i^{\prime}}\right]_{\mathrm{C}}$. Therefore a condition $X \in G$ forces

$$
\forall r\left(r \mathrm { C } \mathbf { a } _ { i } \Rightarrow \varphi \left(\mathbf{x}\lceil J, r) \text { and } r \mathrm{C} \mathbf{a}_{i^{\prime}} \Rightarrow \neg \varphi(\mathbf{x}\lceil J, r))\right.\right. \text {. }
$$

Let $\vartheta=\|X\|$ and $\vartheta^{\prime}=\vartheta \cap J$. Theorem 23 implies (take $\xi_{1}=\{i\}$ and $\left.\xi_{2}=\left\{i^{\prime}\right\}\right)$ that even the weaker condition $Y=X \mid \vartheta^{\prime} \in G$ forces $(*)$.

The automorphism $h$ obviously generates an order automorphism, say $Z \mapsto Z^{\prime}$, of $\mathbb{P}$ onto $\mathbb{P}$. We observe that $Y^{\prime}=Y$ because $h$ is assumed to be the identity on the set $J$ while $\vartheta^{\prime}=\|Y\| \subseteq J$.

We set $G^{\prime}=\left\{Z^{\prime}: Z \in G\right\}$. Then $Y \in G^{\prime}, G^{\prime}$ is $\mathbb{P}$-generic over $\mathfrak{M}$, and moreover, $\mathfrak{M}\left[G^{\prime}\right]=\mathfrak{M}[G]$ because $h \in \mathfrak{M}$. Let $\mathbf{x}^{\prime}=\left\langle\mathbf{a}_{j}^{\prime}: j \in \mathbf{I}\right\rangle \in \mathcal{D}^{\mathbf{I}}$ be defined from $G^{\prime}$ as $\mathbf{x}$ was defined from $G$. Then we have $\mathbf{a}_{h(j)}^{\prime}=\mathbf{a}_{j}$ for all $j$; in particular, (a) $\mathbf{a}_{i^{\prime}}^{\prime}=\mathbf{a}_{i}$, and (b) $\mathbf{x}^{\prime} \uparrow J=\mathbf{x} \uparrow J$.

Since $Y$ forces $(*)$, (b) implies $\neg \varphi(\mathbf{x} \mid J, r)$ in $\mathfrak{N}=\mathfrak{M}\left[G^{\prime}\right]$ for any real $r \in \mathfrak{N}$ satisfying $r \mathbf{C} \mathbf{a}_{i^{\prime}}^{\prime}$ in $\mathfrak{N}$. On the other hand, the same property of $Y$ directly implies $\varphi\left(\mathbf{x}\lceil J, r)\right.$ in $\mathfrak{N}=\mathfrak{M}[G]$ provided $r \mathbf{C} \mathbf{a}_{i}$ in $\mathfrak{N}$, contrary to (a).

Pr o of of The or em 1. In principle, a special choice of a model where $\mathrm{C}$, the equiconstructibility on the reals, neither admits a R-OD enumeration of the equivalence closses by sets of ordinals, nor admits a R-OD pairwise Cinequivalent set of cardinality $\mathfrak{c}$, is not necessary. It turns out that everything that we need in addition to requirements (i) and (ii) (see the beginning of this section) is the three more requirements:

(iii) In $\mathfrak{M}$, I has cardinality $\leq \mathfrak{\aleph}_{2}^{\mathfrak{M}}$ and is not countably cofinal.

(iv) Every proper (i.e. $J \neq \mathbf{I}$ ) initial segment $J \subseteq \mathbf{I}$ (belongs to $\mathfrak{M}$ by (ii) and) satisfies card $J<\operatorname{card} \mathbf{I}$ in $\mathfrak{M}$.

(v) If $J$ is a proper initial segment of $\mathbf{I}$ then there is an order automorphism $h \in \mathfrak{M}$ of $\mathbf{I}$ onto $\mathbf{I}$, equal to the identity on $J$ but not equal to the identity on $\mathbf{I}$. 
Surely a wellordered set I cannot satisfy (v), but we have both nonlinear wellfounded order relations and nonwellordered linear orders $\mathbf{I} \in \mathfrak{M}$ which do satisfy (ii) through (v); see some examples below.

Theorem 36. Suppose (i) through (v). Then it is true in $\mathfrak{N}$ that the equivalence relation $\mathrm{C}$ has $\mathfrak{c}$ equivalence classes and:

- neither has a R-OD enumeration of the equivalence classes by sets of ordinals;

- nor admits a R-OD pairwise C-inequivalent set of cardinality $\mathfrak{c}$.

In addition, $\mathfrak{c}^{\mathfrak{N}}=\aleph_{1}^{\mathfrak{M}}=\aleph_{1}^{\mathfrak{N}}$ in the case $\operatorname{card} \mathbf{I}=\aleph_{1}^{\mathfrak{M}}$ in $\mathfrak{M}$, and $\mathfrak{c}^{\mathfrak{N}}=\aleph_{2}^{\mathfrak{M}}=$ $\aleph_{2}^{\mathfrak{N}}$ in the case $\operatorname{card} \mathbf{I}=\aleph_{2}^{\mathfrak{M}}$ in $\mathfrak{M}$.

This theorem obviously implies Theorem 1, as soon as we are able to realize requirements (ii) through (v) on a partially ordered set $\mathbf{I}$ in a countable model $\mathfrak{M}=\mathrm{V}=\mathrm{L}$.

Proof. We prove the "additional" part of the theorem. The cardinals $\aleph_{1}$ and $\aleph_{2}$ are preserved by Theorem 24 . The reals $\mathbf{a}_{i}(i \in \mathbf{I})$ are pairwise different and $\mathrm{C}$-inequivalent in $\mathfrak{N}$ by Corollary 27 , hence $\mathfrak{c} \geq \operatorname{card} \mathbf{I}$ and $\mathrm{C}$ has at least card $\mathbf{I}$ equivalence classes in $\mathfrak{N}$. On the other hand, we have $\mathfrak{c} \leq \operatorname{card} \boldsymbol{\Xi} \times \aleph_{1}^{\mathfrak{M}}$ in $\mathfrak{N}$ by Theorem 25 , therefore $\mathfrak{c} \leq \operatorname{card} \mathbf{I}$ in $\mathfrak{N}$, whichever cardinality, $\aleph_{1}^{\mathfrak{M}}$ or $\aleph_{2}^{\mathfrak{M}}$, I has in $\mathfrak{M}$. It also follows that $\mathrm{C}$ has exactly $\mathfrak{c}$ equivalence classes in $\mathfrak{N}$.

Thus it remains to prove the "neither" and "nor" statements.

We prove the "nor" part of the theorem. Let a pairwise C-inequivalent set $S$ of reals be defined in $\mathfrak{N}$ by a formula containing ordinals and a real $p \in \mathfrak{N}$ as parameters. It follows from Theorem 30(1) that $J=\mathbf{I}_{p}$ (we have $J \in \mathfrak{M}$ by (ii)) is an initial segment of $\mathbf{I}$ of countable cofinality in $\mathfrak{M}$ and $p \in \mathfrak{M}[\mathbf{x}\lceil J]$. Then $S$ is definable in $\mathfrak{N}$ by a formula containing only $\mathbf{x}\lceil J$ and ordinals as parameters.

We assert that $S \subseteq \mathfrak{M}[\mathbf{x}\rceil J]$. Indeed, let $r \in S$. We have $\mathfrak{M}[r]=\mathfrak{M}[\mathbf{x}\lceil\xi]$ for some $\xi \in \boldsymbol{\Xi}, \xi \subseteq J$, by Theorem 30(1,2). Therefore $r$ is definable in $\mathfrak{N}$ as the unique real $r \in S$ which satisfies the equality $\mathrm{L}[r]=\mathrm{L}[\mathbf{x} \mid \xi]$. To conclude, $r$ is definable in $\mathfrak{N}$ by a formula containing only $\mathbf{x} \uparrow J$, ordinals, and $\mathbf{x} \uparrow \xi$ in the C-invariant form, as parameters. Now $r \in \mathfrak{M}[\mathbf{x}\lceil J]$ by Proposition 34, as required.

It remains to prove that reals in $\mathfrak{M}[\mathbf{x}\lceil J]$ generate less than $\mathfrak{c} \mathfrak{M}$-degrees in $\mathfrak{N}$. It suffices, by Theorem 30(3), to check that $J$ has $<$ card I countably cofinal initial segments in $\mathfrak{M}$.

As $J$ is countably cofinal in $\mathfrak{M}$, it follows from (iii) that $J \neq \mathbf{I}$, hence card $J<\operatorname{card} \mathbf{I}$ in $\mathfrak{M}$ by (iv). We have two cases, by (iii). 
Case 1: $\operatorname{card} \mathbf{I}=\aleph_{1}$ in $\mathfrak{M}$. Then $J$ is countable in $\mathfrak{M}$. The collection IS $_{J}$ of all initial segments of $J$ is a Borel subset of $2^{J}$, hence either $\mathbf{I S}_{J}$ belongs to $\mathfrak{M}$ and is countable in $\mathfrak{M}$, or $\mathbf{I S}_{J} \nsubseteq \mathbb{M}$. However the "or" case is incompatible with (ii).

Case 2: $\operatorname{card} \mathbf{I}=\aleph_{2}$ in $\mathfrak{M}$. Then card $J \leq \aleph_{1}$ in $\mathfrak{M}$, so that $J$ has at most $\aleph_{1}<\operatorname{card} \mathbf{I}=\aleph_{2}$ countably cofinal initial segments in $\mathfrak{M}$ by (i).

We prove the "neither" part of the theorem. It follows from Theorem 30(3) that the map $r \mapsto \mathbf{I}_{r}$ enumerates the C-classes of reals by initial segments of $\mathbf{I}$ (all of them belong to $\mathfrak{M}$ by (ii), therefore we can extract even an enumeration by ordinals) in $\mathfrak{N}$, but we shall see that such an enumeration cannot be R-OD in $\mathfrak{N}$ !

Suppose on the contrary that $U$ is a R-OD enumeration of all C-equivalence classes in $\mathfrak{N}$ by sets of ordinals; in other words, $U$ maps reals to sets of ordinals in such a way that $x \mathrm{C} y$ iff $U(x)=U(y)$ for all reals $x, y$ in $\mathfrak{N}$. Then, as in the proof of the "nor" part, $U$ is definable in $\mathfrak{N}$ by a formula containing ordinals and some $\mathbf{x}\lceil J$ (where $J \in \mathfrak{M}$ is an initial segment of $\mathbf{I}$ satisfying $J \neq \mathbf{I}$ ) as parameters.

We assert that $U(r) \in \mathfrak{M}[\mathbf{x}\lceil J]$ for each real $r \in \mathfrak{N}$. Indeed, as above, there exists $\xi \in \boldsymbol{\Xi}$ such that $\mathfrak{M}[r]=\mathfrak{M}[\mathbf{x}\lceil\xi]$. Then $U(r)$ is definable in $\mathfrak{N}$ as the set of ordinals equal to the value $U\left(r^{\prime}\right)$ for an arbitrary real $r^{\prime}$ such that $\mathrm{L}\left[r^{\prime}\right]=\mathrm{L}[\mathbf{x} \mid \xi]$, hence $U(r) \in \mathfrak{M}[\mathbf{x}\lceil J]$ by Proposition 34 .

Thus each $C$-class is definable in $\mathfrak{N}$ by a formula containing only ordinals and $\mathbf{x}\lceil J$ as parameters. In particular, $\mathbf{x}\lceil J$ plus ordinals is enough to distinguish all C-classes from one other. This leads to a contradiction with Proposition 35. (Indeed, condition (v) provides the existence of $i \in$ $\mathbf{I}$ and an order automorphism $h \in \mathfrak{M}$ of $\mathbf{I}$ such that $h\lceil J$ is the identity but $h(i)=i^{\prime} \neq i$. The C-classes $\left[\mathbf{a}_{i}\right]_{\mathcal{C}}$ and $\left[\mathbf{a}_{i^{\prime}}\right]_{C}$ are different (by Corollary 27) in $\mathfrak{N}$. As demonstrated above, each of the classes is definable in $\mathfrak{N}$ by a formula with only $\mathbf{x}\lceil J$ and ordinals as parameters. But this contradicts Proposition 35: for take $A$ to be any of the two sets, $\left[\mathbf{a}_{i}\right]_{\mathrm{C}}$ or $\left[\mathbf{a}_{i^{\prime}}\right]$ c.)

Particular models. Let $\mathfrak{M}$ be a countable transitive model satisfying the axiom of constructibility, so that (i) is provided. The following examples of the p.o. set $\mathbf{I}$ demonstrate different possibilities of realization of requirements (ii) through (v).

EXAMPLE 1: $I_{1}=\omega_{1}^{\mathfrak{M}} \times\{0,1\}\left(\omega_{1}^{\mathfrak{M}}\right.$ copies of the unordered two-element set $\{0,1\})$, ordered lexicographically. The "symmetries" $\langle\alpha, 0\rangle \leftrightarrow\langle\alpha, 1\rangle$ for large enough ordinals $\alpha$ prove $(\mathrm{v})$. In the extension, $\mathfrak{c}=\aleph_{1}^{\mathfrak{M}}=\aleph_{1}^{\mathfrak{N}}$. (In this case the extension $\mathfrak{N}=\mathfrak{M}[G]$ is in fact the ordinary Sacks $\times$ Sacks countable support iteration of length $\omega_{1}^{\mathfrak{M}}$.) 
EXAmple 2: $I_{2}=\omega_{2}^{\mathfrak{M}} \times\{0,1\}$. Quite similar to the previous one, but we have $\mathfrak{c}=\aleph_{2}^{\mathfrak{M}}=\aleph_{2}^{\mathfrak{N}}$ in the extension. (One gets nothing new taking say $\omega_{3}^{\mathfrak{M}}$, because in this case $\aleph_{2}^{\mathfrak{M}}$ collapses to $\aleph_{1}^{\mathfrak{M}}$ in the extension.)

EXAmple 3: $I_{3}=\omega_{1}^{\mathfrak{M}} \times \mathbb{Z}$ (which means $\omega_{1}^{\mathfrak{M}}$ copies of the integers $\mathbb{Z}=$ $\{\ldots,-2,-1,0,1,2, \ldots\})$, ordered lexicographically. This is a linearly ordered but not wellordered set, so the model cannot be defined as an ordinary Sacks iteration. (v) is provided by shiftings inside a far enough $\mathbb{Z}$-group. We have $\mathfrak{c}=\aleph_{1}^{\mathfrak{M}}=\aleph_{1}^{\mathfrak{N}}$ in the extension.

ExAmple 4: $I_{4}=\omega_{2}^{\mathfrak{M}} \times \mathbb{Z}$. Similar to the previous example, but $\mathfrak{c}=$ $\aleph_{2}^{\mathfrak{M}}=\aleph_{2}^{\mathfrak{N}}$ in the extension.

EXAmple 5: $I_{5}=\omega_{1}^{\mathfrak{M}} \times(\mathbb{Z} \times\{0,1\})$, ordered lexicographically. (As above, the two-element set $\{0,1\}$ is assumed to be unordered, i.e. ordered by the empty order). Thus, from the point of view of $\mathfrak{M}, I_{5}$ is the set of all triples $i=\langle\alpha, z, d\rangle$, where $\alpha<\omega_{1}, z \in \mathbb{Z}$, and $d=0,1$, partially ordered lexicographically, but of course not wellfounded and not linear. To avoid any ambiguity, we stress that $\langle\alpha, z, d\rangle<\left\langle\alpha^{\prime}, z^{\prime}, d^{\prime}\right\rangle$ in $\mathbf{I}$ iff either $\alpha<\alpha^{\prime}$ or $\alpha=\alpha^{\prime} \& z<z^{\prime}$, independently of the values of $d, d^{\prime}$.

EXAmple 6: $I_{6}=\omega_{2}^{\mathfrak{M}} \times(\mathbb{Z} \times\{0,1\})$.

This ends the proof of Theorem 1.

Proof of Theorem 2. Let $\mathbf{I}$ be one of the sets $I_{5}, I_{6}$ henceforth.

(The difference between the two possibilities will be essential only for the computation of $\mathfrak{c}$ in the extension.) The requirements (ii) through (v) are obviously satisfied. Note that the pairs of the form $\{\langle\alpha, z, 0\rangle,\langle\alpha, z, 1\rangle\}$, and only these, are order-incomparable in $\mathbf{I}$.

We keep the notation introduced above. Fix a set $G \subseteq \mathbb{P}, \mathbb{P}$-generic over $\mathfrak{M}$, and consider the extension $\mathfrak{N}=\mathfrak{M}[G]=\mathfrak{M}[\mathbf{x}]=\mathfrak{M}\left[\left\langle\mathbf{a}_{i}: i \in \mathbf{I}\right\rangle\right]$.

The plan is to define, in $\mathfrak{N}$, an uncountable $\Pi_{2}^{1}$ set $W$ such that the relation of equiconstructibility $C$ restricted to $W$ also belongs to $\Pi_{2}^{1}$, prove that $\mathrm{C}\lceil W$ behaves in $\mathfrak{N}$ similarly to the unrestricted $\mathrm{C}$ in the models of the preceding subsection, and finally extend $\mathrm{C} \uparrow W$ to all reals in $\mathfrak{N}$, putting the extended relation to be equality outside $W$.

TheOREM 37. In $\mathfrak{N}$, there exists a $\Pi_{2}^{1}$ set of reals $W$ such that the restricted relation $\mathrm{C} \mid W$ is $\Pi_{2}^{1}$, has $\mathfrak{c}$ equivalence classes, and:

- neither has a R-OD enumeration of the equivalence classes by sets of ordinals;

- nor admits a $R$-OD pairwise inequivalent set of cardinality $\mathfrak{c}$.

In addition, we have $\mathfrak{c}^{\mathfrak{N}}=\aleph_{1}^{\mathfrak{M}}=\aleph_{1}^{\mathfrak{N}}$ in the case $\mathbf{I}=I_{5}$, and $\mathfrak{c}^{\mathfrak{N}}=\aleph_{2}^{\mathfrak{M}}=\aleph_{2}^{\mathfrak{N}}$ in the case $\mathbf{I}=I_{6}$. 
First of all we demonstrate that this theorem implies Theorem $\left.2{ }^{11}\right)$. To extend the relation $\mathrm{C} \uparrow W$ onto all reals define the relation $C^{\prime}$ in $\mathfrak{N}$ by:

$$
x C^{\prime} y \quad \text { iff } \quad(x, y \in W \& x C y) \vee x=y .
$$

The extended relation is a $\Pi_{2}^{1}$ equivalence relation on the reals in $\mathfrak{N}$. Clearly the $C^{\prime}$-classes are the old C-classes of reals in $W$ plus the singletons $\{x\}$, $x \notin W$. Therefore $\mathrm{C}^{\prime}$ cannot admit a R-OD enumeration of the equivalence classes by sets of ordinals since otherwise such an enumeration would be available for $\mathrm{C} \uparrow W$, contrary to Theorem 37 .

Finally, $\mathrm{E}_{0}$ does not R-OD embed in $\mathrm{C}^{\prime}$. Indeed, since $\mathrm{E}_{0}$-classes are countable while the newly added $C^{\prime}$-classes are singletons, such an embedding (a 1-1 function by definition) maps $2^{\omega}$ (the domain of $\mathrm{E}_{0}$ ) into $W$; this yields an uncountable R-OD pairwise C-inequivalent subset of $W$ since $\mathrm{E}_{0}$ admits pairwise inequivalent perfect sets of reals - again contrary to Theorem 37 .

Proof of Theorem 37. We define $W$ as a set of pairs of reals rather than reals themselves, but essentially this does not make a big difference.

Definition. In $\mathfrak{N}, W$ is the set of all pairs $\langle x, y\rangle$ of reals such that, for some ordinal $\alpha\left(\alpha<\omega_{1}^{\mathfrak{M}}\right.$ in the case $\mathbf{I}=I_{5}$ and $\alpha<\omega_{2}^{\mathfrak{M}}$ in the case $\left.\mathbf{I}=I_{6}\right)$ and $z \in \mathbb{Z}$, either $x \mathrm{C} \mathbf{a}_{\alpha z 0}$ and $y \mathrm{C} \mathbf{a}_{\alpha z 1}$, or vice versa $x \mathrm{C} \mathbf{a}_{\alpha z 1}$ and $y \mathrm{C} \mathbf{a}_{\alpha z 0}$.

Lemma 38. In $\mathfrak{N}$, both $W$ and the restriction $\mathrm{C} \mid W$ belong to $\Pi_{2}^{1}$.

(We understand that $\langle x, y\rangle \mathrm{C}\left\langle x^{\prime}, y^{\prime}\right\rangle$ iff $\mathrm{L}[x, y]=\mathrm{L}\left[x^{\prime}, y^{\prime}\right]$. In particular, we have $\langle x, y\rangle \mathrm{C}\langle y, x\rangle$, but $\langle x, y\rangle \mathrm{C}\left\langle x^{\prime}, y^{\prime}\right\rangle$ does not imply $x \mathrm{C} x^{\prime}$ or $y \mathrm{C} y^{\prime}$.)

Proof. We prove that $W=\left\{\langle x, y\rangle \in \mathcal{N}^{2}: x \notin \mathrm{L}[y] \& y \notin \mathrm{L}[x]\right\}$ in $\mathfrak{N}$.

If $\langle x, y\rangle \in W$ then $x \notin \mathrm{L}[y]$ and $y \notin \mathrm{L}[x]$ follow from Lemma 26 since $\langle\alpha, z, 0\rangle$ and $\langle\alpha, z, 1\rangle$ are order-incomparable in $\mathbf{I}$ for all $\alpha$ and $z$.

To prove the converse suppose that reals $x, y$ satisfy $x \notin \mathrm{L}[y]$ and $y \notin \mathrm{L}[x]$ in $\mathfrak{N}$ and show $\langle x, y\rangle \in W$. The initial segments $\mathbf{I}_{x}$ and $\mathbf{I}_{y}$ satisfy $\mathbf{I}_{x} \nsubseteq \mathbf{I}_{y}$ and $\mathbf{I}_{y} \nsubseteq \mathbf{I}_{x}$ by Theorem 30(3). It follows that $\mathbf{I}_{x}$ and $\mathbf{I}_{y}$ have the form $\left[\leq i_{x}\right]$ and $\left[\leq i_{y}\right]$ where $i_{x}=\left\langle\alpha, z, d_{x}\right\rangle$ and $i_{y}=\left\langle\alpha, z, d_{y}\right\rangle$ for one and the same $\alpha, z$ but $\left\{d_{x}, d_{y}\right\}=\{0,1\}$. Then $x \mathbf{C} \mathbf{a}_{i_{x}}$ and $y \mathbf{C} \mathbf{a}_{i_{y}}$ in $\mathfrak{N}$ by Theorem $30(2)$, so $\langle x, y\rangle \in W$.

Thus $W$ is $\Pi_{2}^{1}$. Let us prove that $\mathrm{C}\left\lceil W\right.$ is also $\Pi_{2}^{1}$ in $\mathfrak{N}$.

$\left({ }^{11}\right)$ This argument makes essential use of the fact that an embedding is a bijection by definition. The result becomes false for reductions instead of embeddings. (A reduction is the same as an embedding but not necessarily 1-1.) Indeed, $\mathrm{E}_{0}$ admits a $\Delta_{2}^{1}$ selector in $\mathfrak{N}$; this property of the models we consider is not so easy to prove. It follows that $\mathrm{E}_{0}$ admits a $\Delta_{2}^{1}$ enumeration of the equivalence classes in $\mathfrak{N}$, i.e. a $\Delta_{2}^{1}$ reduction to the equality on $\mathcal{D}$. Taking a perfect subset of $W^{\prime}=\mathcal{D} \backslash W$, we finally get a $\Delta_{2}^{1}$ reduction of $\mathrm{E}_{0}$ to $\mathrm{C}^{\prime}$, more exactly to $C^{\prime}\left\lceil W^{\prime}\right.$. 
It suffices to check that, given pairs $\langle x, y\rangle$ and $\left\langle x^{\prime}, y^{\prime}\right\rangle$ in $W,\langle x, y\rangle \in$ $\mathrm{L}\left[x^{\prime}, y^{\prime}\right]$ iff $\left\langle x^{\prime}, y^{\prime}\right\rangle \notin \mathrm{L}[x]$ in $\mathfrak{N}$.

Let on the contrary $\langle x, y\rangle \in \mathrm{L}\left[x^{\prime}, y^{\prime}\right]$ and $\left\langle x^{\prime}, y^{\prime}\right\rangle \in \mathrm{L}[x]$, so that $y \in$ $\mathrm{L}[x]$ - a contradiction because $x$ and $y$ are incomparable. For the converse, suppose that $\langle x, y\rangle \notin \mathrm{L}\left[x^{\prime}, y^{\prime}\right]$. Since the pairs belong to $W$, one can assume that $\langle x, y\rangle=\left\langle\mathbf{a}_{\alpha z 0}, \mathbf{a}_{\alpha z 1}\right\rangle$ and $\left\langle x^{\prime}, y^{\prime}\right\rangle=\left\langle\mathbf{a}_{\alpha^{\prime} z^{\prime} 0}, \mathbf{a}_{\alpha^{\prime} z^{\prime} 1}\right\rangle$ for some ordinals $\alpha, \alpha^{\prime}$ and integers $z, z^{\prime}$. Since $\langle x, y\rangle \notin \mathrm{L}\left[x^{\prime}, y^{\prime}\right]$, we have $\left\langle\alpha^{\prime}, z^{\prime}\right\rangle\langle\langle\alpha, z\rangle$ lexicographically, and therefore $\left\langle\alpha^{\prime}, z^{\prime}, d^{\prime}\right\rangle\langle\langle\alpha, z, d\rangle$ in $\mathbf{I}$ for any choice of $d, d^{\prime} \in\{0,1\}$. Hence $\left\langle x^{\prime}, y^{\prime}\right\rangle \in \mathrm{L}[x]$ in $\mathfrak{N}$ by Lemma 22 , as required.

After we have established the class $\Pi_{2}^{1}$ of $W$ and $\mathrm{C} \uparrow W$, the remainder of the proof of Theorem 37 can be carried out similarly to the proof of Theorem 36 above. In particular, the same reasoning proves the "additional" assertion, as well as the fact that $\mathrm{C}$ has $\mathfrak{c}$ classes on $W$. But the "neither" and "nor" assertions need some care.

We prove the "nor" part of Theorem 37 . Assume on the contrary that, in $\mathfrak{N}, S \subseteq W$ is a pairwise C-inequivalent R-OD subset of $W$ of cardinality $\mathfrak{c}$. (Recall that $W$ consists of pairs of reals.) Then, in $\mathfrak{N}$, the set $S^{\prime}=\{x: \exists y(\langle x, y\rangle \in S)\}$ is a pairwise C-inequivalent R-OD set of reals of cardinality $\mathfrak{c}$ - contrary to Theorem 36 .

We prove the "neither" part. Suppose on the contrary that, in $\mathfrak{N}, U$ enumerates $(\mathrm{C} \uparrow W)$-equivalence classes by subsets of an ordinal $\gamma$; thus $U$ maps $W$ into $\mathcal{P}(\gamma)$ so that $U(x, y)=U\left(x^{\prime}, y^{\prime}\right)$ iff $\langle x, y\rangle \mathrm{C}\left\langle x^{\prime}, y^{\prime}\right\rangle$.

Obviously if both $\langle x, y\rangle$ and $\left\langle x, y^{\prime}\right\rangle$ belong to $W$ then $y \mathrm{C} y^{\prime}$, so we have $U(x, y)=U\left(x, y^{\prime}\right)$. Thus one can define, for each real $x \in W^{\prime}=\{x:$ $\exists y(\langle x, y\rangle \in W)\}, U^{\prime}(x)=U(x, y)$ for any $y$ satisfying $\langle x, y\rangle \in W$.

Notice that $W^{\prime}$ is the set of all reals $x \in \mathfrak{N}$ such that $x \mathbf{C} \mathbf{a}_{i}$ in $\mathfrak{N}$ for some $i=\langle\alpha, z, d\rangle \in \mathbf{I}$, in particular, $W^{\prime}$ is a C-invariant set.

It is not completely true that $U^{\prime}$ enumerates C-classes of reals in $W^{\prime}$. $x$ C $x^{\prime}$ still implies $U^{\prime}(x)=U^{\prime}\left(x^{\prime}\right)$, but not conversely. But the following is true: if $U^{\prime}(x)=U^{\prime}\left(x^{\prime}\right)$ then there exist $\alpha\left(\alpha<\omega_{1}^{\mathfrak{M}}\right.$ in the case $\mathbf{I}=I_{5}$ and $\alpha<\omega_{2}^{\mathfrak{M}}$ in the case $\left.\mathbf{I}=I_{6}\right)$ and $z \in \mathbb{Z}$ such that each of the reals $x, x^{\prime}$ is C-equivalent to one of $\mathbf{a}_{\alpha z 0}, \mathbf{a}_{\alpha z 1}$, independently of each other.

(Thus $U^{\prime}$ is an enumeration of the $\mathrm{C}^{+}$-equivalence classes, where the equivalence $\mathrm{C}^{+}$, in addition to $\mathrm{C}$, glues each pair $\mathbf{a}_{\alpha z 0}, \mathbf{a}_{\alpha z 1}$ in one class. This "amalgamation" of classes makes the symmetries $\langle\alpha, z, 0\rangle \leftrightarrow\langle\alpha, z, 1\rangle$ useless, but fortunately we still have shiftings inside $\mathbb{Z}$-groups.)

Note that $U^{\prime}$ is definable in $\mathfrak{N}$ by a formula containing, as parameters, only ordinals and some $\mathbf{x} \uparrow J$, where $J \in \mathfrak{M}$ is a countably cofinal initial segment of $\mathbf{I}$ not equal to $\mathbf{I}$ (see the proof of Theorem 36). Then $U^{\prime}(x) \in$ $\mathrm{L}\left[\mathbf{x}\lceil J]\right.$ in $\mathfrak{N}$ for all reals $x \in W^{\prime}$, again as in the proof of Theorem 36 . 
Since $J \neq \mathbf{I}$, there exists an ordinal $\alpha\left(\alpha<\omega_{1}^{\mathfrak{M}}\right.$ in the case $\mathbf{I}=I_{5}$ and $\alpha<\omega_{2}^{\mathfrak{M}}$ in the case $\left.\mathbf{I}=I_{6}\right)$ such that $\langle\alpha, z, d\rangle \notin J$ for all $z$ and $d$. In particular, neither $i=\langle\alpha, 7,0\rangle$ nor $i^{\prime}=\langle\alpha, 8,0\rangle$ is a member of $J$.

Define an order automorphism $h$ of $\mathbf{I}$ by $h(\langle\alpha, z, d\rangle)=\langle\alpha, z+1, d\rangle$ for $d=0,1$, all integers $z$, and this particular $\alpha$, and $h\left(\left\langle\alpha^{\prime}, z, d\right\rangle\right)=\left\langle\alpha^{\prime}, z, d\right\rangle$ whenever $\alpha^{\prime} \neq \alpha$. Then $h \in \mathfrak{M}, h(i)=i^{\prime}$, but $h \uparrow J$ is the identity.

To accomplish the proof of the "neither" part of Theorem 37, it now suffices to reproduce the very end of the proof of Theorem 36, taking e.g. the set $A=\left[\mathbf{a}_{\alpha 70}\right]_{\mathrm{E}} \cup\left[\mathbf{a}_{\alpha 71}\right]_{\mathrm{E}}$ in the application of Proposition 35 .

This also ends the proof of Theorem 2.

Acknowledgements. I would like to thank S. D. Friedman, M. Gitik, G. Hjorth, A. S. Kechris, P. Koepke, A. W. Miller, J. Steprāns for useful discussions and/or interesting information on the equivalence relations and iterated Sacks forcing, and the referee for some improvements. This paper was written partially during my visits to IPM (Tehran), Max Planck Institute (Bonn), and University of Wuppertal in 1995-1996 and I am grateful to the people there, especially M. J. A. Larijani, the president of IPM, P. Koepke, at Bonn, and M. Reeken at UW, for the support and hospitality.

\section{References}

[1] J. E. Baumgartner and R. Laver, Iterated perfect set forcing, Ann. Math. Logic 17 (1979), 271-288.

[2] S. D. Friedman and B. Velickovic, Nonstandard models and analytic equivalence relations, Proc. Amer. Math. Soc., to appear.

[3] M. Groszek, $\omega_{1}^{*}$ as an initial segment of the c-degrees, J. Symbolic Logic 59 (1994), 956-976.

[4] M. Groszek and T. Jech, Generalized iteration of forcing, Trans. Amer. Math. Soc. 324 (1991), 1-26.

[5] L. A. Harrington, A. S. Kechris and A. Louveau, A Glimm-Effros dichotomy for Borel equivalence relations, J. Amer. Math. Soc. 3 (1990), 903-928.

[6] G. Hjorth, Thin equivalence relations and effective decompositions, J. Symbolic Logic 58 (1993), 1153-1164.

[7] -, A dichotomy for the definable universe, ibid. 60 (1995), 1199-1207.

[8] - A remark on $\Pi_{1}^{1}$ equivalence relations, note, 1994.

[9] G. Hjorth and A. S. Kechris, Analytic equivalence relations and Ulm-type classifications, J. Symbolic Logic 60 (1995), 1273-1300.

[10] V. Kanovei, The cardinality of the set of Vitali equivalence classes, Math. Notes 49 (1991), 370-374.

[11] - An Ulm-type classification theorem for equivalence relations in Solovay model, J. Symbolic Logic 62 (1997), to appear.

[12] -, Ulm classification of analytic equivalence relations in generic universes, Math. Logic Quart. 44 (1998), to appear. 
[13] A. S. Kechris, Topology and descriptive set theory, Topology Appl. 58 (1994), 195-222.

[14] —, Classical Descriptive Set Theory, Springer, 1995.

[15] N. Lusin, Sur les ensembles analytiques, Fund. Math. 10 (1927), 1-95.

[16] W. Sier piński, L'axiome de M. Zermelo et son rôle dans la théorie des ensembles et l'analyse, Bull. Internat. Acad. Sci. Lettres Sér. A Sci. Math. 1918, 97-152.

[17] J. Silver, Counting the number of equivalence classes of Borel and coanalytic equivalence relations, Ann. Math. Logic 18 (1980), 1-28.

Department of Mathematics

Moscow Transport Engineering Institute (MIIT)

Obraztsova 15

Moscow 101475, Russia

E-mail: kanovei@mech.math.msu.su

Received 15 January 1996;

in revised form 20 January 1997 